\title{
Open WDVV Equations and Virasoro Constraints
}

\author{
Alexey Basalaev ${ }^{1} \cdot$ Alexandr Buryak $^{2}$
}

Received: 13 February 2019 / Revised: 4 June 2019 / Accepted: 10 June 2019 / Published online: 24 June 2019 (c) The Author(s) 2019

\begin{abstract}
In their fundamental work, Dubrovin and Zhang, generalizing the Virasoro equations for the genus 0 Gromov-Witten invariants, proved the Virasoro equations for a descendent potential in genus 0 of an arbitrary conformal Frobenius manifold. More recently, a remarkable system of partial differential equations, called the open WDVV equations, appeared in the work of Horev and Solomon. This system controls the genus 0 open Gromov-Witten invariants. In our paper, for an arbitrary solution of the open WDVV equations, satisfying a certain homogeneity condition, we construct a descendent potential in genus 0 and prove an open analog of the Virasoro equations. We also present conjectural open Virasoro equations in all genera and discuss some examples.
\end{abstract}

Keywords Riemann surface $\cdot$ Moduli space $\cdot$ WDVV equations

\section{Contents}

1 Introduction . . . . . . . . . . . . . . . . . . . . . . . . . 146

2 Virasoro Constraints for Frobenius Manifolds . . . . . . . . . . . . . . . . . 150

2.1 Descendent Potential . . . . . . . . . . . . . . . . . . . . . . . . . . . . 151

2.2 Virasoro Constraints . . . . . . . . . . . . . . . . . . . . . . . . . . 152

3 Open Virasoro Constraints . . . . . . . . . . . . . . . . . . . . . . . . . 154

4 Virasoro Type Constraints for Flat F-manifolds . . . . . . . . . . . . . . . . . . . . . 157

4.1 Flat F-manifolds . . . . . . . . . . . . . . . . . . . . . . . . . . . . . . . . 157

4.2 Extensions of Flat F-manifolds and the Open WDVV Equations $\ldots \ldots \ldots \ldots \ldots$

4.3 Calibration of a Flat F-manifold . . . . . . . . . . . . . . . . . . . . 159

4.3 .1 General Case . . . . . . . . . . . . . . . . . . . . . . . . . . . . . . . . . . . 159

Dedicated to Rafail Kalmanovich Gordin on the occasion of his 70th birthday.

Alexandr Buryak

a.buryak@leeds.ac.uk

Alexey Basalaev

a.basalaev@skoltech.ru

1 Skolkovo Institute of Science and Technology, Nobelya Ulitsa 3, Moscow 121205, Russian

Federation

2 School of Mathematics, University of Leeds, Leeds LS2 9JT, UK 
4.3 .2 Conformal Case . . . . . . . . . . . . . . . . . . . . . . . 160

4.3.3 Calibrations of Extensions of Flat F-manifolds . . . . . . . . . . . . . . . . . 162

4.4 Descendent Vector Potentials of a Flat F-manifold . . . . . . . . . . . . . . . . . . . . . . 164

4.5 Virasoro Constraints . . . . . . . . . . . . . . . . . . . . . . 167

4.6 Proof of Theorem $3.4 \ldots \ldots \ldots \ldots \ldots$. . . . . . . . . . . . . . . . . . 173

5 Open Virasoro Constraints in All Genera . . . . . . . . . . . . . . . . . . . . . . . . 174

6 Examples of Solutions of the Open WDVV Equations . . . . . . . . . . . . . . . . . . . 182

6.1 Extended $r$-Spin Theory . . . . . . . . . . . . . . . . . . . . . . . . . . . 182

6.2 Solutions Given by the Canonical Coordinates . . . . . . . . . . . . . . . . . . . 183

6.3 Open Gromov-Witten Theory of $\mathbb{P}^{1} \ldots \ldots \ldots$. . . . . . . . . . . . . . . . . . . . 184

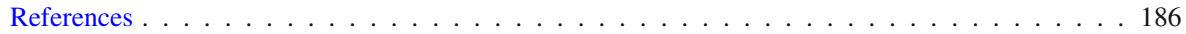

\section{Introduction}

The WDVV equations, also called the associativity equations, is a system of nonlinear partial differential equations for one function, depending on a finite number of variables. Let $N \geq 1$ and $\eta=\left(\eta_{\alpha \beta}\right)$ be an $N \times N$ symmetric non-degenerate matrix with complex coefficients. The WDVV equations is the following system of PDEs for a function $F\left(t^{1}, \ldots, t^{N}\right)$ defined on some open subset $M \subset \mathbb{C}^{N}$ :

$$
\frac{\partial^{3} F}{\partial t^{\alpha} \partial t^{\beta} \partial t^{\mu}} \eta^{\mu \nu} \frac{\partial^{3} F}{\partial t^{\nu} \partial t^{\gamma} \partial t^{\delta}}=\frac{\partial^{3} F}{\partial t^{\delta} \partial t^{\beta} \partial t^{\mu}} \eta^{\mu \nu} \frac{\partial^{3} F}{\partial t^{\nu} \partial t^{\gamma} \partial t^{\alpha}}, \quad 1 \leq \alpha, \beta, \gamma, \delta \leq N
$$

where $\left(\eta^{\alpha \beta}\right):=\eta^{-1}$ and we use the convention of sum over repeated Greek indices. Suppose that the function $F$ satisfies the additional assumption $\frac{\partial^{3} F}{\partial t^{1} \partial t^{\alpha} \partial t^{\beta}}=\eta_{\alpha \beta}$. Then the function $F$ defines a structure of Frobenius manifold on $M$ and is also called the Frobenius manifold potential. Such a structure appears in different areas of mathematics, including the singularity theory and curve counting theories in algebraic geometry (Gromov-Witten theory, Fan-Jarvis-Ruan-Witten theory). A systematic study of Frobenius manifolds was first done by Dubrovin $(1996,1999)$.

Consider formal variables $t_{p}^{\alpha}, 1 \leq \alpha \leq N, p \geq 0$, where we identify $t_{0}^{\alpha}=t^{\alpha}$. There is a natural way to associate to the function $F$ a descendent potential $\mathcal{F}$, which is a function of the variables $t_{p}^{\alpha}$, such that the difference $\left.\mathcal{F}\right|_{t_{\geq 1}^{*}=0}-F$ is at most quadratic in the variables $t^{1}, \ldots, t^{N}$ and the following equations are satisfied:

$$
\frac{\partial^{3} \mathcal{F}}{\partial t_{a+1}^{\alpha} t_{b}^{\beta} \partial t_{c}^{\gamma}}=\frac{\partial^{2} \mathcal{F}}{\partial t_{a}^{\alpha} \partial t_{0}^{\mu}} \eta^{\mu \nu} \frac{\partial^{3} \mathcal{F}}{\partial t_{0}^{\nu} \partial t_{b}^{\beta} \partial t_{c}^{\gamma}}, \quad 1 \leq \alpha, \beta, \gamma \leq N, \quad a, b, c \geq 0
$$

These equations are called the topological recursion relations (TRR). In GromovWitten theory, where the function $F$ is the generating series of intersection numbers on the moduli space of maps from a Riemann surface of genus 0 to a target variety, a natural descendent potential $\mathcal{F}$ is given by the generating series of intersection numbers with the Chern classes of certain line bundles over the moduli space. Note that the system of Eq. (1.2) can be equivalently written as 


$$
d\left(\frac{\partial^{2} \mathcal{F}}{\partial t_{a+1}^{\alpha} \partial t_{b}^{\beta}}\right)=\frac{\partial^{2} \mathcal{F}}{\partial t_{a}^{\alpha} \partial t_{0}^{\mu}} \eta^{\mu v} d\left(\frac{\partial^{2} \mathcal{F}}{\partial t_{0}^{\nu} \partial t_{b}^{\beta}}\right), \quad 1 \leq \alpha, \beta \leq N, \quad a, b \geq 0,
$$

where $d(\cdot)$ denotes the full differential.

Let $\varepsilon$ be a formal variable and $\widetilde{t}_{p}^{\alpha}:=t_{p}^{\alpha}-\delta^{\alpha, 1} \delta_{p, 1}$. If our Frobenius manifold is conformal, meaning that the function $F$ satisfies a certain homogeneity condition, then in Dubrovin and Zhang (1999) the authors constructed differential operators $L_{m}$, $m \geq-1$, of the form

$$
\begin{aligned}
L_{m}= & \sum_{p, q \geq 0}\left(\varepsilon^{2} a_{m}^{\alpha, p ; \beta, q} \frac{\partial^{2}}{\partial t_{p}^{\alpha} \partial t_{q}^{\beta}}+b_{m ; \alpha, p}^{\beta, q} \widetilde{t}_{p}^{\alpha} \frac{\partial}{\partial t_{q}^{\beta}}+\varepsilon^{-2} c_{m ; \alpha, p ; \beta, q} \widetilde{t}_{p}^{\alpha} \widetilde{t}_{q}^{\beta}\right) \\
& + \text { const } \cdot \delta_{m, 0}, \quad a_{m}^{\alpha, p ; \beta, q}, b_{m ; \alpha, p}^{\beta, q}, c_{m ; \alpha, p ; \beta, q} \in \mathbb{C},
\end{aligned}
$$

satisfying the commutation relations

$$
\left[L_{i}, L_{j}\right]=(i-j) L_{i+j}, \quad i, j \geq-1,
$$

and such that the following equations, called the Virasoro constraints, are satisfied:

$$
\operatorname{Coef}_{\varepsilon^{-2}}\left(\frac{L_{m} e^{\varepsilon^{-2} \mathcal{F}}}{e^{\varepsilon^{-2} \mathcal{F}}}\right)=0, \quad m \geq-1
$$

We recall the details in Sect. 2.

In Gromov-Witten theory, for each $g \geq 0$ one defines the generating series $\mathcal{F}_{g}\left(t_{*}^{*}\right)$ of intersection numbers on the moduli space of maps from a Riemann surface of genus $g$ to a target variety, $\mathcal{F}_{0}=\mathcal{F}$. The Virasoro conjecture says that the following equations are satisfied:

$$
L_{m} e^{\sum_{g \geq 0} \varepsilon^{2 g-2} \mathcal{F}_{g}}=0, \quad m \geq-1
$$

One can see that Eq. (1.4) is the genus 0 part of Eq. (1.5). The Virasoro conjecture is proved in a wide class of cases, but is still open in the whole generality.

More recently, a remarkable system of PDEs, extending the WDVV Eq. (1.1), appeared in the literature. Let $s$ be a formal variable. The open WDVV equations are the following PDEs for a function $F^{o}\left(t^{1}, \ldots, t^{N}, s\right)$ :

$$
\begin{aligned}
& \frac{\partial^{3} F}{\partial t^{\alpha} \partial t^{\beta} \partial t^{\mu}} \eta^{\mu \nu} \frac{\partial^{2} F^{o}}{\partial t^{\nu} \partial t^{\gamma}}+\frac{\partial^{2} F^{o}}{\partial t^{\alpha} \partial t^{\beta}} \frac{\partial^{2} F^{o}}{\partial s \partial t^{\gamma}} \\
& =\frac{\partial^{3} F}{\partial t^{\gamma} \partial t^{\beta} \partial t^{\mu}} \eta^{\mu \nu} \frac{\partial^{2} F^{o}}{\partial t^{\nu} \partial t^{\alpha}}+\frac{\partial^{2} F^{o}}{\partial t^{\gamma} \partial t^{\beta}} \frac{\partial^{2} F^{o}}{\partial s \partial t^{\alpha}}, \quad 1 \leq \alpha, \beta, \gamma \leq N,
\end{aligned}
$$




$$
\begin{gathered}
\frac{\partial^{3} F}{\partial t^{\alpha} \partial t^{\beta} \partial t^{\mu}} \eta^{\mu \nu} \frac{\partial^{2} F^{o}}{\partial t^{\nu} \partial s}+\frac{\partial^{2} F^{o}}{\partial t^{\alpha} \partial t^{\beta}} \frac{\partial^{2} F^{o}}{\partial s^{2}} \\
=\frac{\partial^{2} F^{o}}{\partial s \partial t^{\beta}} \frac{\partial^{2} F^{o}}{\partial s \partial t^{\alpha}}, \quad 1 \leq \alpha, \beta \leq N .
\end{gathered}
$$

These equations first appeared in Horev and Solomon (2012, Theorem 2.7) in the context of open Gromov-Witten theory. The open WDVV equations also appeared in the works (Pandharipande et al. 2014; Buryak et al. 2018, 2019). The solutions of Eqs. (1.6), (1.7), considered in these works, also satisfy the additional condition

$$
\frac{\partial^{2} F^{o}}{\partial t^{1} \partial t^{\alpha}}=0, \quad \frac{\partial^{2} F^{o}}{\partial t^{1} \partial s}=1 .
$$

Remark 1.1 The works (Pandharipande et al. 2014; Buryak et al. 2018, 2019 don't mention the open WDVV explicitly, but, as it is explained in Buryak (2018, Section 4), the open WDVV equations follow immediately from the open TRR equations (Pandharipande et al. 2014, Theorem 1.5), (Buryak et al. 2018, Theorem 4.1), (Buryak et al. 2019, Lemma 3.6).

There is an open analog of Eq. (1.3). Let $s_{p}, p \geq 0$, be formal variables, where we identify $s_{0}=s$. The open topological recursion relations are the following PDEs for a function $\mathcal{F}^{o}$, depending on the variables $t_{p}^{\alpha}$ and $s_{p}$, and such that the difference $\left.\mathcal{F}^{o}\right|_{\substack{t_{\geq 1}^{*}=0 \\ s \geq 1}}-F^{o}$ is at most linear in the variables $t^{1}, \ldots, t^{N}$ and $s$ :

$$
\begin{gathered}
d\left(\frac{\partial \mathcal{F}^{o}}{\partial t_{p+1}^{\alpha}}\right)=\frac{\partial^{2} \mathcal{F}}{\partial t_{p}^{\alpha} \partial t_{0}^{\mu}} \eta^{\mu \nu} d\left(\frac{\partial \mathcal{F}^{o}}{\partial t_{0}^{\nu}}\right)+\frac{\partial \mathcal{F}^{o}}{\partial t_{p}^{\alpha}} d\left(\frac{\partial \mathcal{F}^{o}}{\partial s}\right), \quad 1 \leq \alpha \leq N, \quad p \geq 0 \\
d\left(\frac{\partial \mathcal{F}^{o}}{\partial s_{p+1}}\right)=\frac{\partial \mathcal{F}^{o}}{\partial s_{p}} d\left(\frac{\partial \mathcal{F}^{o}}{\partial s}\right), \quad p \geq 0
\end{gathered}
$$

As we already mentioned in Remark 1.1, these equations appeared in the works (Pandharipande et al. 2014; Buryak et al. 2018, 2019).

The simplest Frobenius manifold has dimension 1 and the potential $F=F^{\mathrm{pt}}=$ $\frac{\left(t^{1}\right)^{3}}{6}$. A natural descendent potential $\mathcal{F}^{\mathrm{pt}}$, associated to it, is given by the generating series of the integrals of monomials in the psi-classes over the moduli space of stable curves of genus 0. Here "pt" means "point", because such integrals can be considered as the Gromov-Witten invariants of a point. One can easily see that the function $F^{o}=F^{\mathrm{pt}, o}=t^{1} s+\frac{s^{3}}{6}$ satisfies the open WDVV equations and condition (1.8). In Pandharipande et al. (2014) the authors, using the intersection theory on the moduli space of stable pointed disks, constructed a solution $\mathcal{F}^{\mathrm{pt}, o}$ of the open TRR Eqs. (1.9), (1.10). Moreover, they introduced the operators 


$$
\begin{aligned}
\mathcal{L}_{m}^{\mathrm{pt}}:= & L_{m}^{\mathrm{pt}}+\varepsilon^{-1} \delta_{m,-1} s+\left(\sum_{i \geq 0} \frac{(i+m+1) !}{i !} s_{i} \frac{\partial}{\partial s_{m+i}}+\delta_{m, 0} \frac{3}{4}\right) \\
& +\varepsilon \frac{3(m+1) !}{4} \frac{\partial}{\partial s_{m-1}}, \quad m \geq-1,
\end{aligned}
$$

where $L_{m}^{\mathrm{pt}}$ are the Virasoro operators for our Frobenius manifold, and proved the equations (Pandharipande et al. 2014, Theorem 1.1)

$$
\operatorname{Coef}_{\varepsilon^{-1}}\left(\frac{\mathcal{L}_{m}^{\mathrm{pt}} e^{\varepsilon^{-2} \mathcal{F}^{\mathrm{pt}}+\varepsilon^{-1} \mathcal{F}^{\mathrm{pt}, o}}}{e^{\varepsilon^{-2} \mathcal{F}^{\mathrm{pt}}+\varepsilon^{-1} \mathcal{F}^{\mathrm{pt}, o}}}\right)=0, \quad m \geq-1,
$$

which they called the open Virasoro constraints.

Remark 1.2 Strictly speaking, in Pandharipande et al. (2014) the authors constructed a function $\mathcal{F}^{\mathrm{PST}}$, related to our function $\mathcal{F}^{\mathrm{pt}, o}$ by $\mathcal{F}^{\mathrm{PST}}=\left.\mathcal{F}^{\mathrm{pt}, o}\right|_{s_{\geq 1}=0}$. The function $\mathcal{F}^{\mathrm{pt}, o}$ can be reconstructed from the function $\mathcal{F}^{\mathrm{PST}}$, using the differential equations $\frac{\partial \mathcal{F}^{\mathrm{pt}, o}}{\partial s_{n}}=\frac{1}{(n+1) !}\left(\frac{\partial \mathcal{F}^{\mathrm{pt}, o}}{\partial s}\right)^{n+1}$. The Virasoro equations, proved in Pandharipande et al. (2014), look as

$$
\begin{aligned}
& \operatorname{Coef}_{\varepsilon^{-1}}\left(\frac{\mathcal{L}_{m}^{\mathrm{PST}} e^{\varepsilon^{-2} \mathcal{F}^{\mathrm{pt}}+\varepsilon^{-1} \mathcal{F}^{\mathrm{PST}}}}{e^{\varepsilon^{-2} \mathcal{F}^{\mathrm{pt}}+\varepsilon^{-1} \mathcal{F}^{\mathrm{PST}}}}\right)=0, \\
& \mathcal{L}_{m}^{\mathrm{PST}}:=L_{m}^{\mathrm{pt}}+\varepsilon^{m} s \frac{\partial^{m+1}}{\partial s^{m+1}}+\frac{3 m+3}{4} \varepsilon^{m} \frac{\partial^{m}}{\partial s^{m}}, \quad m \geq-1 .
\end{aligned}
$$

The fact, that Eqs. (1.12) and (1.13) are equivalent, was noticed in Buryak (2016, Section 5.2).

In our paper, we generalize formula (1.12) for an arbitrary conformal Frobenius manifold and a solution of the open WDVV equations. We consider an arbitrary conformal Frobenius manifold, an associated descendent potential $\mathcal{F}$ and the Virasoro operators $L_{m}, m \geq-1$. Let $F^{o}$ be a solution of the open WDVV equations, satisfying condition (1.8) and a certain homogeneity condition, that we will describe later. We will construct a solution $\mathcal{F}^{o}$ of the open TRR Eqs. (1.9), (1.10) and differential operators $\mathcal{L}_{m}, m \geq-1$, of the form

$$
\begin{aligned}
\mathcal{L}_{m}= & L_{m}+\varepsilon^{-1}\left(\delta_{m,-1} s+\sum_{p \geq 0} d_{m ; \alpha, p} \widetilde{t}_{p}^{\alpha}\right) \\
& +\left(\sum_{i \geq 0} \frac{(i+m+1) !}{i !} s_{i} \frac{\partial}{\partial s_{m+i}}+\sum_{p, q \geq 0} e_{m ; \alpha, p}^{q} \widetilde{t}_{p}^{\alpha} \frac{\partial}{\partial s_{q}}+\delta_{m, 0} \frac{3}{4}\right) \\
& +\varepsilon\left(\sum_{p \geq 0} f_{m}^{\alpha, p} \frac{\partial}{\partial t_{p}^{\alpha}}+\frac{3(m+1) !}{4} \frac{\partial}{\partial s_{m-1}}\right)
\end{aligned}
$$




$$
+\varepsilon^{2} \sum_{p, q \geq 0} g_{m}^{\alpha, p ; q} \frac{\partial^{2}}{\partial t_{p}^{\alpha} \partial s_{q}}, \quad d_{m ; \alpha, p}, e_{m ; \alpha, p}^{q}, f_{m}^{\alpha, p}, g_{m}^{\alpha, p ; q} \in \mathbb{C}
$$

such that the equations

$$
\operatorname{Coef}_{\varepsilon^{-1}}\left(\frac{\mathcal{L}_{m} e^{\varepsilon^{-2} \mathcal{F}+\varepsilon^{-1} \mathcal{F}^{o}}}{e^{\varepsilon^{-2} \mathcal{F}+\varepsilon^{-1} \mathcal{F} o}}\right)=0, \quad m \geq-1,
$$

hold. The details are given in Sect. 3, with the main result, formulated in Theorem 3.4.

It occurs that, given a function $F=F\left(t^{1}, \ldots, t^{N}\right)$, defining a Frobenius manifold and a solution $F^{o}$ of the open WDVV equations, satisfying (1.8), the $(N+1)$-tuple of functions $\left(\eta^{1 \mu} \frac{\partial F}{\partial t^{\mu}}, \ldots, \eta^{N \mu} \frac{\partial F}{\partial t^{\mu}}, F^{o}\right)$ forms a vector potential of a flat $F$-manifold. This was observed by Paolo Rossi. We say that this flat F-manifold extends the Frobenius manifold given. In Sect. 4 we prove Virasoro type constraints for flat F-manifolds and derive Theorem 3.4 as a special case of this result.

Let us return to the particular case, considered in the paper (Pandharipande et al. 2014). The construction of the intersection theory on the moduli space of stable pointed disks, given there, can be generalized to higher genera. This has been announced by Solomon and Tessler, some of the details of their construction are presented in Tessler (2015). As a result, one gets a sequence of functions $\mathcal{F}_{0}^{\mathrm{pt}, o}=$ $\mathcal{F}^{\mathrm{pt}, o}, \mathcal{F}_{1}^{\mathrm{pt}, o}, \mathcal{F}_{2}^{\mathrm{pt}, o}, \ldots$, and already in [PST14] the authors conjectured that the following equations should hold:

$$
\mathcal{L}_{m}^{\mathrm{pt}} e^{\sum_{g \geq 0} \varepsilon^{2 g-2} \mathcal{F}_{g}^{\mathrm{pt}}+\sum_{g \geq 0} \varepsilon^{g-1} \mathcal{F}_{g}^{\mathrm{pt}, o}}=0, \quad m \geq-1,
$$

where $\mathcal{F}_{g}^{\mathrm{pt}}$ is the generating series of the intersection numbers of psi-classes on the moduli space of curves of genus $g$. This conjecture was proved in Buryak and Tessler (2017).

In Sect. 5 we discuss a conjectural generalization of Eq. (1.15) for an arbitrary conformal Frobenius manifold and a solution of the open WDVV equations.

Finally, in Sect. 6 we present examples of solutions of the open WDVV equations, for which our main result can be applied. In a forthcoming paper (Basalaev and Buryak 2019) we will discuss solutions of the open WDVV equations for the Coxeter groups.

\section{Virasoro Constraints for Frobenius Manifolds}

In this section we review the construction of a descendent potential associated to a solution of the WDVV equations and recall the Virasoro constraints.

Let us fix $N \geq 1$ and let $M$ be a simply connected open neighbourhood of a point $\left(t_{\text {orig }}^{1}, \ldots, t_{\text {orig }}^{N}\right) \in \mathbb{C}^{N}$. Denote by $\mathcal{O}$ the sheaf of analytic functions on $\mathbb{C}^{N}$. Consider a solution $F \in \mathcal{O}(M)$ of the WDVV Eq. (1.1), satisfying $\frac{\partial^{3} F}{\partial t^{1} \partial t^{\alpha} \partial t^{\beta}}=\eta_{\alpha \beta}$. In order to include the case, when $F$ is a formal power series, in our considerations, we allow $M$ to be a formal neighbourhood of $\left(t_{\text {orig }}^{1}, \ldots, t_{\text {orig }}^{N}\right) \in \mathbb{C}^{N}$ meaning that $\mathcal{O}(M)$ denotes in this case the ring of formal power series in the variables $\left(t^{\alpha}-t_{\text {orig }}^{\alpha}\right)$. 


\subsection{Descendent Potential}

In order to construct a descendent potential, one has to choose an additional structure, called a calibration of the Frobenius manifold. Denote

$$
c_{\beta \gamma}^{\alpha}:=\eta^{\alpha \mu} \frac{\partial^{3} F}{\partial t^{\mu} \partial t^{\beta} \partial t^{\gamma}} .
$$

A calibration is a collection of functions $\Omega_{\beta, d}^{\alpha, 0} \in \mathcal{O}(M), 1 \leq \alpha, \beta \leq N, d \geq-1$, satisfying the following properties:

$$
\begin{aligned}
& \Omega_{\beta,-1}^{\alpha, 0}=\delta_{\beta}^{\alpha}, \\
& \frac{\partial \Omega_{\beta, d}^{\alpha, 0}}{\partial t^{\gamma}}=c_{\gamma \mu}^{\alpha} \Omega_{\beta, d-1}^{\mu, 0}, \quad d \geq 0, \\
& \sum_{\substack{p+q=d \\
p, q \geq-1}}^{\alpha \geq 1)^{p+1} \Omega_{\mu, p}^{\alpha, 0} \eta^{\mu \nu} \Omega_{v, q}^{\beta, 0}=0, \quad d \geq-1 .}
\end{aligned}
$$

The space of all calibrations is non-empty and is parameterized by elements $G(z) \in$ $\operatorname{Mat}_{N, N}(\mathbb{C})[[z]]$, satisfying $G(0)=\operatorname{Id}$ and $G(-z) \eta^{-1} G^{T}(z)=\eta^{-1}$ (Dubrovin 1999, Lemma 2.2 and Exercise 2.8).

Let us choose a calibration. One can immediately see that $\frac{\partial \Omega_{1,0}^{\alpha, 0}}{\partial t^{\beta}}=\delta_{\beta}^{\alpha}$, which implies that $\Omega_{1,0}^{\alpha, 0}-t^{\alpha}$ is a constant. Let us make the change of coordinates $t^{\alpha} \mapsto \Omega_{1,0}^{\alpha, 0}$, so that we have now $\Omega_{1,0}^{\alpha, 0}=t^{\alpha}$.

Let $v^{1}, \ldots, v^{N}$ be formal variables and consider the system of partial differential equations

$$
\frac{\partial v^{\alpha}}{\partial t_{d}^{\beta}}=\partial_{x}\left(\left.\Omega_{\beta, d}^{\alpha, 0}\right|_{t \gamma=v^{\gamma}}\right), \quad 1 \leq \alpha, \beta \leq N, \quad d \geq 0
$$

called the principal hierarchy. We see that the equations for the flow $\frac{\partial}{\partial t_{0}^{1}}$ look as $\frac{\partial v^{\alpha}}{\partial t_{0}^{1}}=v_{x}^{\alpha}$. This allows to identify $t_{0}^{1}=x$. Denote by $\left(v^{\text {top }}\right)^{\alpha} \in \mathcal{O}(M)\left[\left[t_{\geq 1}^{*}\right]\right]$ the solution of the principal hierarchy specified by the initial condition

$$
\left.\left(v^{\text {top }}\right)^{\alpha}\right|_{t_{d}^{\beta}=\delta^{\beta, 1} \delta_{d, 0} x}=\delta^{\alpha, 1} x .
$$

We have $\left.\left(v^{\text {top }}\right)^{\alpha}\right|_{t_{\geq 1}^{*}=0}=t_{0}^{\alpha}$.

Define functions $\Omega_{\alpha, p ; \beta, q} \in \mathcal{O}(M)$ and $\Omega_{\alpha, p ; \beta, q}^{\text {top }} \in \mathcal{O}(M)\left[\left[t_{\geq 1}^{*}\right]\right], p, q \geq 0$, by

$$
\Omega_{\alpha, p ; \beta, q}:=\sum_{i=0}^{q}(-1)^{q-i} \Omega_{\alpha, p+q-i}^{\mu, 0} \eta_{\mu \nu} \Omega_{\beta, i-1}^{\nu, 0}, \quad \Omega_{\alpha, p ; \beta, q}^{\text {top }}:=\left.\Omega_{\alpha, p ; \beta, q}\right|_{t^{\gamma}=\left(v^{\text {top }}\right)^{\gamma}} .
$$


The descendent potential corresponding to the Frobenius manifold together with the chosen calibration is defined by

$$
\mathcal{F}:=\frac{1}{2} \sum_{p, q \geq 0} \widetilde{t}_{p}^{\alpha} \widetilde{t}_{q}^{\beta} \Omega_{\alpha, p ; \beta, q}^{\mathrm{top}} \in \mathcal{O}(M)\left[\left[t_{\geq 1}^{*}\right]\right],
$$

where we recall that $\widetilde{t}_{p}^{\alpha}=t_{p}^{\alpha}-\delta^{\alpha, 1} \delta_{p, 1}$ (Dubrovin and Zhang 1999, Section 3). The difference $\left.\mathcal{F}\right|_{t_{\geq 1}^{*}=0}-F$ is at most quadratic in the variables $t^{1}, \ldots, t^{N}$ and the function $\mathcal{F}$ satisfies Eq. (1.2) together with the equation

$$
\sum_{p \geq 0} \widetilde{t}_{p+1}^{\alpha} \frac{\partial \mathcal{F}}{\partial t_{p}^{\alpha}}+\frac{1}{2} \eta_{\alpha \beta} t^{\alpha} t^{\beta}=0
$$

which is called the string equation (Dubrovin and Zhang 1999, Sections 3, 4).

Remark 2.1 Strictly speaking, in Dubrovin (1999) and Dubrovin and Zhang (1999) the authors consider the case of a conformal Frobenius manifold, but one can easily see that the results, discussed in this section, together with their proofs presented in Dubrovin (1999), Dubrovin and Zhang (1999), hold for all not necessarily conformal Frobenius manifolds. We have borrowed the term "calibration" from the paper (Dubrovin et al. 2016).

\subsection{Virasoro Constraints}

The Frobenius manifold is said to be conformal if there exists a vector field $E$ of the form

$$
E=\underbrace{\left(\left(1-q^{\gamma}\right) t^{\gamma}+r^{\gamma}\right)}_{=: E^{\gamma}} \frac{\partial}{\partial t^{\gamma}}, \quad q^{\gamma}, r^{\gamma} \in \mathbb{C}, \quad q^{1}=0,
$$

satisfying

$$
L_{E} F=(3-\delta) F+\frac{1}{2} A_{\alpha \beta} t^{\alpha} t^{\beta}+B_{\alpha} t^{\alpha}+C, \quad \text { for some } \delta, A_{\alpha \beta}, B_{\alpha}, C \in \mathbb{C} \text {. }
$$

The number $\delta$ is often called the conformal dimension. Denote

$$
\mu^{\alpha}:=q^{\alpha}-\frac{\delta}{2}, \quad \mu:=\operatorname{diag}\left(\mu^{1}, \ldots, \mu^{N}\right)
$$

In the conformal case there exists a calibration, satisfying the property

$$
E^{\theta} \frac{\partial}{\partial t^{\theta}} \Omega_{\beta, d}^{\alpha, 0}=\left(d+1+\mu^{\beta}-\mu^{\alpha}\right) \Omega_{\beta, d}^{\alpha, 0}+\sum_{i=1}^{d+1} \Omega_{\mu, d-i}^{\alpha, 0}\left(R_{i}\right)_{\beta}^{\mu}, \quad d \geq-1,
$$


for some matrices $R_{n}, n \geq 1$, satisfying

$$
\left[\mu, R_{n}\right]=n R_{n}, \quad \eta R_{n} \eta^{-1}=(-1)^{n-1} R_{n}^{T} .
$$

One can actually see that the matrices $R_{n}$ are determined uniquely by the functions $\Omega_{\beta, d}^{\alpha, 0}$. Note that only finitely many of the matrices $R_{n}$ are non-zero. The space of all calibrations, satisfying property (2.2), can be explicitly described, see (Dubrovin 1999, Section 2). A calibration of a conformal Frobenius manifold will always be assumed to satisfy property (2.2).

Let us choose a calibration of our conformal Frobenius manifold and denote

$$
R:=\sum_{i \geq 1} R_{i}
$$

For an arbitrary $N \times N$ matrix $A=\left(A_{\beta}^{\alpha}\right)$ define matrices $P_{m}(A, R), m \geq-1$, by the following recursion relation:

$$
\begin{aligned}
P_{-1}(A, R) & =\mathrm{Id} \\
P_{m+1}(A, R) & =R P_{m}(A, R)+P_{m}(A, R)\left(A+m+\frac{1}{2}\right), \quad m \geq-1 .
\end{aligned}
$$

Alternatively, the matrix $P_{m}(A, R)$ can be defined by

$$
P_{m}(A, R)=: \prod_{i=0}^{m}\left(R+A+i-\frac{1}{2}\right):
$$

where the symbol :: means that, when we take the product, we should place all $R$ 's to the left of all $A$ 's. Given an integer $p$, define a matrix $[A]_{p}$ by

$$
\left([A]_{p}\right)_{\beta}^{\alpha}:= \begin{cases}A_{\beta}^{\alpha}, & \text { if } q^{\alpha}-q^{\beta}=p \\ 0, & \text { otherwise. }\end{cases}
$$

The Virasoro operators $L_{m}, m \geq-1$, of our conformal Frobenius manifold are given by

$$
\begin{aligned}
L_{m}:= & \frac{\varepsilon^{-2}}{2} \sum_{d_{1}, d_{2} \geq 0}(-1)^{d_{1}}\left(\left[P_{m}\left(\mu+d_{2}+1, R\right)\right]_{m+d_{1}+d_{2}+1}\right)_{\beta}^{\mu} \eta_{\alpha \mu} \widetilde{t}_{d_{1}}^{\alpha} \widetilde{t}_{d_{2}}^{\beta} \\
& +\sum_{\substack{d \geq 0 \\
0 \leq k \leq m+d}}\left(\left[P_{m}(\mu+d+1, R)\right]_{m+d-k}\right)_{\alpha}^{\beta} \widetilde{t}_{d}^{\alpha} \frac{\partial}{\partial t_{k}^{\beta}} \\
& +\frac{\varepsilon^{2}}{2} \sum_{\substack{d_{1}+d_{2} \leq m-1 \\
d_{1}, d_{2} \geq 0}}(-1)^{d_{2}+1}\left(\left[P_{m}\left(\mu-d_{2}, R\right)\right]_{m-1-d_{1}-d_{2}}\right)_{\mu}^{\alpha} \eta^{\mu \beta} \frac{\partial^{2}}{\partial t_{d_{1}}^{\alpha} \partial t_{d_{2}}^{\beta}}
\end{aligned}
$$




$$
+\delta_{m, 0} \frac{1}{4} \operatorname{tr}\left(\frac{1}{4}-\mu^{2}\right), \quad m \geq-1,
$$

and Eq. (1.4) hold (Dubrovin and Zhang 1999, page 428).

Remark 2.2 One can easily see that the last term $\delta_{m, 0} \frac{1}{4} \operatorname{tr}\left(\frac{1}{4}-\mu^{2}\right)$ in the expression for the Virasoro operators $L_{m}$ doesn't play a role in Eq. (1.4). However, this term plays a role in the Virasoro constraints in all genera (1.5). We discuss it in more details in Sect. 5.

\section{Open Virasoro Constraints}

Here we present our construction of an open descendent potential associated to a solution of the open WDVV equations and present our main result- the open Virasoro constraints, given in Theorem 3.4.

Consider a conformal Frobenius manifold $M$ together with its calibration from Sect. 2.2. Let $U$ be a simply connected open neighbourhood of a point $s_{\text {orig }} \in \mathbb{C}$. Suppose that a function $F^{o}\left(t^{1}, \ldots, t^{N}, s\right) \in \mathcal{O}(M \times U)$ satisfies the open WDVV Eqs. (1.6), (1.7) and condition (1.8). Let us assume that the function $F^{o}$ satisfies the homogeneity condition

$$
\begin{aligned}
& E^{\gamma} \frac{\partial F^{o}}{\partial t^{\gamma}}+\underbrace{\left(\frac{1-\delta}{2} s+r^{N+1}\right)}_{=: E^{N+1}} \frac{\partial F^{o}}{\partial s} \\
& =\frac{3-\delta}{2} F^{o}+D_{\alpha} t^{\alpha}+\widetilde{D} s+E, \text { for some } r^{N+1}, D_{\alpha}, \widetilde{D}, E \in \mathbb{C} .
\end{aligned}
$$

This condition holds in the examples, considered in the papers (Horev and Solomon 2012; Pandharipande et al. 2014; Buryak et al. 2018, 2019). Actually, in these examples the constant $r^{N+1}$ is zero, but this is not needed in our considerations.

In order to construct an open descendent potential $\mathcal{F}^{o}$ we need an additional structure, similar to a calibration of a Frobenius manifold. It will be convenient for us to denote the variable $s_{d}$ by $t_{d}^{N+1}$. We adopt the conventions

$$
\Omega_{N+1, d}^{\alpha, 0}:=0, \quad 1 \leq \alpha \leq N, \quad d \geq-1, \quad \mu^{N+1}:=\frac{1}{2}
$$

and define a diagonal $(N+1) \times(N+1)$ matrix $\tilde{\mu}$ by

$$
\tilde{\mu}:=\operatorname{diag}\left(\mu^{1}, \ldots, \mu^{N+1}\right) .
$$


Definition 3.1 A calibration of the function $F^{o}$ is a sequence of functions $\Phi_{\beta, d} \in$ $\mathcal{O}(M \times U), 1 \leq \beta \leq N+1, d \geq-1$, satisfying the properties

$$
\begin{aligned}
\Phi_{\beta,-1} & =\delta_{\beta, N+1}, \\
\frac{\partial \Phi_{\beta, d}}{\partial t^{\gamma}} & =\sum_{1 \leq \mu \leq N} \frac{\partial^{2} F^{o}}{\partial t^{\gamma} \partial t^{\mu}} \Omega_{\beta, d-1}^{\mu, 0}+\frac{\partial^{2} F^{o}}{\partial t^{\gamma} \partial t^{N+1}} \Phi_{\beta, d-1}, \quad d \geq 0, \\
E^{\mu} \frac{\partial \Phi_{\beta, d}}{\partial t^{\mu}} & =\left(d+\frac{1}{2}+\mu^{\beta}\right) \Phi_{\beta, d}+\sum_{i=1}^{d+1} \Phi_{\mu, d-i}\left(\widetilde{R}_{i}\right)_{\beta}^{\mu}, \quad d \geq-1,
\end{aligned}
$$

for some $(N+1) \times(N+1)$ matrices $\widetilde{R}_{n}, n \geq 1$, satisfying

$$
\left(\widetilde{R}_{n}\right)_{\beta}^{\alpha}=\left\{\begin{array}{ll}
\left(R_{n}\right)_{\beta}^{\alpha}, & \text { if } 1 \leq \alpha, \beta \leq N, \\
0, & \text { if } \beta=N+1,
\end{array} \quad\left[\widetilde{\mu}, \widetilde{R}_{n}\right]=n \widetilde{R}_{n}\right.
$$

One can easily see that, for a calibration of the function $F^{o}$, matrices $\widetilde{R}_{n}$ are uniquely determined by the functions $\Phi_{\beta, d}$.

Lemma 3.2 The space of calibrations of the function $F^{o}$ is non-empty.

The proof of the lemma will be given in Sect. 4.3.3.

Consider a calibration of the function $F^{o}$. One can easily see that $\frac{\partial \Phi_{1,0}}{\partial t^{\gamma}}=\delta_{\gamma, N+1}$, which implies that $\Phi_{1,0}-t^{N+1}$ is a constant. Let us make the change of coordinates $t^{N+1} \mapsto \Phi_{1,0}$, so that we have now $\Phi_{1,0}=t^{N+1}$.

Let $\widetilde{v}^{1}, \widetilde{v}^{2}, \ldots, \widetilde{v}^{N+1}$ be formal variables and consider the system of partial differential equations

$$
\begin{cases}\frac{\partial \widetilde{v}^{\alpha}}{\partial t_{d}^{\beta}}=\partial_{x}\left(\left.\Omega_{\beta, d}^{\alpha, 0}\right|_{t^{\gamma}=\widetilde{v}^{\gamma}}\right), & 1 \leq \alpha \leq N, \quad 1 \leq \beta \leq N+1, \quad d \geq 0, \\ \frac{\partial \widetilde{v}^{N+1}}{\partial t_{d}^{\beta}}=\partial_{x}\left(\left.\Phi_{\beta, d}\right|_{t^{\gamma}=\widetilde{v}^{\gamma}}\right), & 1 \leq \beta \leq N+1, \quad d \geq 0 .\end{cases}
$$

Let $\left(\widetilde{v}^{\text {top }}\right)^{\alpha}$ be the solution, specified by the initial condition

$$
\left.\left(\widetilde{v}^{\mathrm{top}}\right)^{\alpha}\right|_{t_{d}^{\beta}=\delta^{\beta, 1} \delta_{d, 0} x}=\delta^{\alpha, 1} x
$$

Clearly, $\left(\widetilde{v}^{\text {top }}\right)^{\alpha}=\left(v^{\text {top }}\right)^{\alpha}$, for $1 \leq \alpha \leq N$. Define

$$
\Phi_{\beta, d}^{\mathrm{top}}:=\left.\Phi_{\beta, d}\right|_{t^{\gamma}=\left(\widetilde{v}^{\mathrm{top}}\right)^{\gamma}} .
$$

Then we define the open descendent potential $\mathcal{F}^{o}$ by

$$
\mathcal{F}^{o}:=\sum_{d \geq 0} \widetilde{t}_{d}^{\alpha} \Phi_{\alpha, d}^{\mathrm{top}}
$$


Lemma 3.3 The function $\mathcal{F}^{o}$ satisfies Eqs. (1.9), (1.10) and the difference $\left.\mathcal{F}^{o}\right|_{t_{\geq 1}^{*}=0}-$ $F^{o}$ is at most linear in the variables $t^{1}, \ldots, t^{N}$ and $s$.

We will prove the lemma in Sect. 4.4.

Let $L_{m}, m \geq-1$, be the Virasoro operators for our conformal Frobenius manifold and define operators $\mathcal{L}_{m}, m \geq-1$, by

$$
\begin{aligned}
\mathcal{L}_{m}:= & L_{m}+\varepsilon^{-1} \sum_{d \geq 0} \sum_{1 \leq \alpha \leq N+1}\left(\left[P_{m}(\tilde{\mu}+d+1, \widetilde{R})\right]_{m+d+1}\right)_{\alpha}^{N+1} \tilde{t}_{d}^{\alpha} \\
& +\left(\sum_{\substack{d \geq 0 \\
0 \leq k \leq m+d}} \sum_{1 \leq \alpha \leq N+1}\left(\left[P_{m}(\tilde{\mu}+d+1, \widetilde{R})\right]_{m+d-k}\right)_{\alpha}^{N+1} \tilde{t}_{d}^{\alpha} \frac{\partial}{\partial t_{k}^{N+1}}+\delta_{m, 0} \frac{3}{4}\right) \\
& +\varepsilon\left(\sum_{0 \leq k \leq m} \sum_{1 \leq \alpha, \mu \leq N}(-1)^{k+1}\left(\left[P_{m}(\tilde{\mu}-k, \widetilde{R})\right]_{m-k}\right)_{\alpha}^{N+1} \eta^{\alpha \mu} \frac{\partial}{\partial t_{k}^{\mu}}+\frac{3(m+1) !}{4} \frac{\partial}{\partial t_{m-1}^{N+1}}\right) \\
& +\varepsilon^{2} \sum_{\substack{d_{1}, d_{2} \geq 0 \\
d_{1}+d_{2} \leq m-1}} \sum_{1 \leq \alpha, \mu \leq N}(-1)^{d_{2}+1}\left(\left[P_{m}\left(\tilde{\mu}-d_{2}, \widetilde{R}\right)\right]_{m-1-d_{1}-d_{2}}\right)_{\alpha}^{N+1} \eta^{\alpha \mu} \frac{\partial^{2}}{\partial t_{d_{1}}^{N+1} \partial t_{d_{2}}^{\mu}} .
\end{aligned}
$$

Theorem 3.4 We have

$$
\operatorname{Coef}_{\varepsilon^{-1}}\left(\frac{\mathcal{L}_{m} e^{\varepsilon^{-2} \mathcal{F}+\varepsilon^{-1} \mathcal{F}^{o}}}{e^{\varepsilon^{-2} \mathcal{F}+\varepsilon^{-1} \mathcal{F} o}}\right)=0, \quad m \geq-1 .
$$

Remark 3.5 Since $\widetilde{R}_{N+1}^{\alpha}=0$, we have

$$
\begin{array}{ll}
\left(\left[P_{m}(\tilde{\mu}+d+1, \widetilde{R})\right]_{m+d+1}\right)_{N+1}^{N+1}=\delta_{m,-1} \delta_{d, 0}, & m \geq-1, d \geq 0, \\
\left(\left[P_{m}(\widetilde{\mu}+d+1, \widetilde{R})\right]_{m+d-k}\right)_{N+1}^{N+1}=\delta_{k, m+d} \frac{(d+m+1) !}{d !}, & m \geq-1, d \geq 0,0 \leq k \leq m+d .
\end{array}
$$

Therefore, the operators $\mathcal{L}_{m}$, given by (3.5), have the form (1.14).

Remark 3.6 One can easily see that the expression $\frac{\mathcal{L}_{m} e^{\varepsilon^{-2} \mathcal{F}+\varepsilon^{-1} \mathcal{F}^{o}}}{e^{\varepsilon^{-2} \mathcal{F}+\varepsilon^{-1} \mathcal{F}^{o}}}$ has the form

$$
\frac{\mathcal{L}_{m} e^{\varepsilon^{-2} \mathcal{F}+\varepsilon^{-1} \mathcal{F}^{o}}}{e^{\varepsilon^{-2} \mathcal{F}+\varepsilon^{-1} \mathcal{F} o}}=\sum_{i \geq-2} \varepsilon^{i} f_{i}\left(t_{*}^{*}\right)
$$

for some functions $f_{i}$ depending on the variables $t_{p}^{\alpha}, 1 \leq \alpha \leq N+1, p \geq 0$. Note that the vanishing of the function $f_{-2}$ is equivalent to the Virasoro equations for the descendent potential $\mathcal{F}$, 


$$
f_{-2}=\operatorname{Coef}_{\varepsilon^{-2}}\left(\frac{L_{m} e^{\varepsilon^{-2} \mathcal{F}}}{e^{\varepsilon^{-2} \mathcal{F}}}\right)=0 .
$$

Remark 3.7 One can see that the terms $\delta_{m, 0} \frac{3}{4}$ and $\varepsilon \frac{3(m+1) !}{4} \frac{\partial}{\partial t_{m-1}^{N+1}}$ in the expression for the operator $\mathcal{L}_{m}$ don't play a role in Eq. (3.6). However, they play a role in our conjectural open Virasoro constraints in all genera, which we discuss in Sect. 5.

In the next section we derive Virasoro type equations for flat F-manifolds and then get Theorem 3.4 as a special case of this result.

\section{Virasoro Type Constraints for Flat F-manifolds}

In this section we recall the definition of a flat F-manifold and show how such an object can be associated to a solution of the open WDVV equations. We then present a construction of descendent vector potentials, corresponding to a flat F-manifold, and prove Virasoro type constraints for them. The open Virasoro constraints from Theorem 3.4 are derived as a corollary of this result.

\subsection{Flat F-manifolds}

Here we recall the definition and the main properties of flat F-manifolds. We refer a reader to the papers (Manin 2005; Arsie and Lorenzoni 2018) for more details.

Definition 4.1 A flat F-manifold $(M, \nabla, \circ)$ is the datum of an analytic manifold $M$, an analytic connection $\nabla$ in the tangent bundle $T M$, an algebra structure $\left(T_{p} M, \circ\right)$ with unit $e$ on each tangent space, analytically depending on the point $p \in M$, such that the one-parameter family of connections $\nabla+z \circ$ is flat and torsionless for any $z \in \mathbb{C}$, and $\nabla e=0$.

From the flatness and the torsionlessness of $\nabla+z \circ$ one can deduce the commutativity and the associativity of the algebras $\left(T_{p} M, \circ\right)$. Moreover, if one choses flat coordinates $t^{\alpha}, 1 \leq \alpha \leq N, N=\operatorname{dim} M$, for the connection $\nabla$, with $e=\frac{\partial}{\partial t^{1}}$, then it is easy to see that locally there exist analytic functions $F^{\alpha}\left(t^{1}, \ldots, t^{N}\right), 1 \leq \alpha \leq N$, such that the second derivatives

$$
c_{\beta \gamma}^{\alpha}=\frac{\partial^{2} F^{\alpha}}{\partial t^{\beta} \partial t^{\gamma}}
$$

give the structure constants of the algebras $\left(T_{p} M, \circ\right)$,

$$
\frac{\partial}{\partial t^{\beta}} \circ \frac{\partial}{\partial t^{\gamma}}=c_{\beta \gamma}^{\alpha} \frac{\partial}{\partial t^{\alpha}}
$$

From the associativity of the algebras $\left(T_{p} M, \circ\right)$ and the fact that the vector $\frac{\partial}{\partial t^{1}}$ is the unit it follows that 


$$
\begin{gathered}
\frac{\partial^{2} F^{\alpha}}{\partial t^{1} \partial t^{\beta}}=\delta_{\beta}^{\alpha}, \quad 1 \leq \alpha, \beta \leq N, \\
\frac{\partial^{2} F^{\alpha}}{\partial t^{\beta} \partial t^{\mu}} \frac{\partial^{2} F^{\mu}}{\partial t^{\gamma} \partial t^{\delta}}=\frac{\partial^{2} F^{\alpha}}{\partial t^{\gamma} \partial t^{\mu}} \frac{\partial^{2} F^{\mu}}{\partial t^{\beta} \partial t^{\delta}}, \quad 1 \leq \alpha, \beta, \gamma, \delta \leq N .
\end{gathered}
$$

The $N$-tuple of functions $\left(F^{1}, \ldots, F^{N}\right)$ is called the vector potential of the flat Fmanifold.

Conversely, if $M$ is an open subset of $\mathbb{C}^{N}$ and $F^{1}, \ldots, F^{N} \in \mathcal{O}(M)$ are functions, satisfying Eqs. (4.2) and (4.3), then these functions define a flat F-manifold $(M, \nabla, \circ)$ with the connection $\nabla$, given by $\nabla_{\frac{\partial}{\partial t^{\alpha}}} \frac{\partial}{\partial t^{\beta}}=0$, and the multiplication $\circ$, given by the structure constants (4.1).

A flat F-manifold, given by a vector potential $\left(F^{1}, \ldots, F^{N}\right)$, is called conformal, if there exists a vector field of the form (2.1) such that

$$
E^{\mu} \frac{\partial F^{\alpha}}{\partial t^{\mu}}=\left(2-q^{\alpha}\right) F^{\alpha}+A_{\beta}^{\alpha} t^{\beta}+B^{\alpha}, \text { for some } A_{\beta}^{\alpha}, B^{\alpha} \in \mathbb{C} \text {. }
$$

Remark 4.2 A Frobenius manifold with a potential $F$ and a metric $\eta$ defines the flat F-manifold with the vector potential $F^{\alpha}=\eta^{\alpha \mu} \frac{\partial F}{\partial t^{\mu}}$. If the Frobenius manifold is conformal, then the associated flat F-manifold is also conformal. This follows from the property

$$
\left(q^{\alpha}+q^{\beta}-\delta\right) \eta_{\alpha \beta}=0
$$

A point $p \in M$ of an $N$-dimensional flat F-manifold $(M, \nabla, \circ)$ is called semisimple if $T_{p} M$ has a basis of idempotents $\pi_{1}, \ldots, \pi_{N}$, satisfying $\pi_{k} \circ \pi_{l}=\delta_{k, l} \pi_{k}$. Moreover, locally around such a point one can choose coordinates $u^{i}$ such that $\frac{\partial}{\partial u^{k}} \circ \frac{\partial}{\partial u^{l}}=\delta_{k, l} \frac{\partial}{\partial u^{k}}$. These coordinates are called the canonical coordinates. In particular, this means that the semisimplicity is an open property of a point. The flat F-manifold $M$ is called semisimple, if a generic point of $M$ is semisimple.

Remark 4.3 In the semisimple case, a conformal flat F-manifold is a special case of a bi-flat F-manifold, see (Arsie and Lorenzoni 2017, Theorem 4.4).

\subsection{Extensions of Flat F-manifolds and the Open WDVV Equations}

Consider a flat F-manifold structure, given by a vector potential $\left(F^{1}, \ldots, F^{N+1}\right)$ on an open subset $M \times U \in \mathbb{C}^{N+1}$, where $M$ and $U$ are open subsets of $\mathbb{C}^{N}$ and $\mathbb{C}$, respectively. Suppose that the functions $F^{1}, \ldots, F^{N}$ don't depend on the variable $t^{N+1}$, varying in $U$. Then the functions $F^{1}, \ldots, F^{N}$ satisfy Eq. (4.3) and, thus, define a flat F-manifold structure on $M$. In this case we call the flat F-manifold structure on $M \times U$ an extension of a flat F-manifold structure on $M$.

Consider the flat F-manifold, associated to a Frobenius manifold, given by a potential $F\left(t^{1}, \ldots, t^{N}\right) \in \mathcal{O}(M)$ and a metric $\eta, F^{\alpha}=\eta^{\alpha \mu} \frac{\partial F}{\partial t^{\mu}}, 1 \leq \alpha \leq N$. It is easy to check that a function $F^{o}\left(t^{1}, \ldots, t^{N}, s\right) \in \mathcal{O}(M \times U)$ satisfies Eqs. (1.6), (1.7) and (1.8) if and only if the $(N+1)$-tuple $\left(F^{1}, \ldots, F^{N}, F^{o}\right)$ is a vector potential of a 
flat F-manifold. Recall that here we identify $s=t^{N+1}$. This defines a correspondence between solutions of the open WDVV equations, satisfying property (1.8), and flat F-manifolds, extending the Frobenius manifold given. This observation belongs to Paolo Rossi.

\subsection{Calibration of a Flat F-manifold}

In this section we introduce the notion of a calibration of a flat F-manifold. This is done analogously to the case of Frobenius manifolds.

\subsubsection{General Case}

Let $M$ be a simply connected open neighbourhood of a point $\left(t_{\text {orig }}^{1}, \ldots, t_{\text {orig }}^{N}\right) \in \mathbb{C}^{N}$ and consider a flat F-manifold structure on $M$ given by a vector potential $\left(F^{1}, \ldots, F^{N}\right)$, $F^{\alpha} \in \mathcal{O}(M)$. A calibration of our flat F-manifold is a collection of functions $\Omega_{\beta, 0}^{\alpha, d} \in$ $\mathcal{O}(M), 1 \leq \alpha, \beta \leq N, d \geq-1$, satisfying $\Omega_{\beta, 0}^{\alpha,-1}=\delta_{\beta}^{\alpha}$ and the property

$$
\frac{\partial \Omega_{\beta, 0}^{\alpha, d}}{\partial t^{\gamma}}=c_{\gamma \beta}^{\mu} \Omega_{\mu, 0}^{\alpha, d-1}, \quad d \geq 0
$$

Let us describe the space of all calibrations of our flat F-manifold. Denote by $\bar{\nabla}$ the family of connections, depending on a formal parameter $z$, given by

$$
\bar{\nabla}_{X} Y:=\nabla_{X} Y+z X \circ Y
$$

where $X$ and $Y$ are vector fields on $M$. Then Eq. (4.5) is equivalent to the flatness, with respect to $\bar{\nabla}$, of the 1 -forms

$$
\left(\sum_{d \geq 0} \Omega_{\beta, 0}^{\alpha, d-1} z^{d}\right) d t^{\beta}, \quad 1 \leq \alpha \leq N
$$

From the flatness of the connection $\bar{\nabla}$ it follows that a calibration of our flat F-manifold exists. In order to describe the whole space of calibrations, introduce $N \times N$ matrices $\Omega_{0}^{d}, d \geq-1$, by $\left(\Omega_{0}^{d}\right)_{\beta}^{\alpha}:=\Omega_{\beta, 0}^{\alpha, d}$. Then Eq. (4.5) can be written as

$$
\frac{\partial}{\partial t^{\gamma}}\left(\sum_{d \geq 0} \Omega_{0}^{d-1} z^{d}\right)=z\left(\sum_{d \geq 0} \Omega_{0}^{d-1} z^{d}\right) C_{\gamma},
$$

where $C_{\gamma}:=\left(c_{\gamma \beta}^{\alpha}\right)$. From this it becomes clear that the generating series $\sum_{d \geq 0} \Omega_{0}^{d-1} z^{d}$ is determined uniquely up to a transformation of the form 


$$
\sum_{d \geq 0} \Omega_{0}^{d-1} z^{d} \mapsto G(z)\left(\sum_{d \geq 0} \Omega_{0}^{d-1} z^{d}\right), \quad G(z) \in \operatorname{Mat}_{N, N}(\mathbb{C})[[z]], \quad G(0)=\mathrm{Id} .
$$

Let us introduce matrices $\Omega_{d}^{0}=\left(\Omega_{\beta, d}^{\alpha, 0}\right), d \geq 0$, by the equation

$$
\left(\mathrm{Id}+\sum_{d \geq 1}(-1)^{d} \Omega_{d-1}^{0} z^{d}\right)\left(\mathrm{Id}+\sum_{d \geq 1} \Omega_{0}^{d-1} z^{d}\right)=\mathrm{Id} .
$$

By definition, we put $\Omega_{-1}^{0}:=$ Id. From Eq. (4.6) we get

$$
\frac{\partial \Omega_{d}^{0}}{\partial t^{\gamma}}=C_{\gamma} \Omega_{d-1}^{0}, \quad d \geq 0
$$

\subsubsection{Conformal Case}

Suppose now that our flat F-manifold is conformal. Introduce a diagonal matrix $Q$ by

$$
Q:=\operatorname{diag}\left(q^{1}, \ldots, q^{N}\right)
$$

Proposition 4.4 There exists a calibration such that

$$
E^{\theta} \frac{\partial \Omega_{0}^{d}}{\partial t^{\theta}}=(d+1) \Omega_{0}^{d}+\left[\Omega_{0}^{d}, Q\right]+\sum_{i=1}^{d+1}(-1)^{i-1} R_{i} \Omega_{0}^{d-i}, \quad d \geq-1
$$

for some matrices $R_{n}, n \geq 1$, satisfying $\left[Q, R_{n}\right]=n R_{n}$.

Proof Similarly to the work (Dubrovin 1999, pages 310,312), the proposition is proved by considering a certain flat connection on $M \times \mathbb{C}^{*}$.

Introduce a family of connections $\widetilde{\nabla}^{\lambda}$, depending on a complex parameter $\lambda$, on $M \times$ $\mathbb{C}^{*}$ by

$$
\begin{aligned}
\widetilde{\nabla}_{X}^{\lambda} Y:=\nabla_{X} Y+z X \circ Y, & \widetilde{\nabla}_{\frac{\partial}{\partial z}}^{\lambda} Y:=\frac{\partial Y}{\partial z}+E \circ Y+\frac{\lambda-Q}{z} Y, \\
\widetilde{\nabla}_{X}^{\lambda} \frac{\partial}{\partial z}=\widetilde{\nabla}_{\frac{\partial}{\partial z}}^{\lambda} \frac{\partial}{\partial z}:=0, &
\end{aligned}
$$

where $X$ and $Y$ are vector fields on $M \times \mathbb{C}^{*}$ having zero component along $\mathbb{C}^{*}$.

Remark 4.5 Note that for the flat F-manifold, associated to a conformal Frobenius manifold $M$, the connection $\widetilde{\nabla}^{\frac{\delta}{2}}$ coincides with the flat connection $\widetilde{\nabla}$ on $M \times \mathbb{C}^{*}$ from the paper (Dubrovin 1999, page 310).

Lemma 4.6 The connection $\widetilde{\nabla}^{\lambda}$ is flat. 
Proof Direct computation analogous to the one from (Dubrovin 1999, proof of Proposition 2.1).

A differential form $\zeta_{\alpha}\left(t^{*}, z\right) d t^{\alpha}$ on $M \times \mathbb{C}^{*}$ is flat with respect to the connection $\widetilde{\nabla}^{\lambda}$ if and only if the following equations are satisfied:

$$
\begin{aligned}
& \frac{\partial \zeta_{\alpha}}{\partial t^{\beta}}=z c_{\beta \alpha}^{\gamma} \zeta_{\gamma}, \\
& \frac{\partial \zeta_{\alpha}}{\partial z}=\mathcal{U}_{\alpha}^{\gamma} \zeta_{\gamma}+\frac{\lambda-q^{\alpha}}{z} \zeta_{\alpha},
\end{aligned}
$$

where $\mathcal{U}_{\beta}^{\alpha}:=E^{\mu} c_{\mu \beta}^{\alpha}$. Denote by $\zeta$ the row vector $\left(\zeta_{1}, \ldots, \zeta_{N}\right)$, then the last two equations can be written as

$$
\begin{gathered}
\frac{\partial \zeta}{\partial t^{\beta}}=z \zeta C_{\beta}, \\
\frac{\partial \zeta}{\partial z}=\zeta\left(\mathcal{U}+\frac{\lambda-Q}{z}\right),
\end{gathered}
$$

where $\mathcal{U}:=\left(\mathcal{U}_{\beta}^{\alpha}\right)$.

Let us construct a certain matrix solution of the system (4.10), (4.11). We first consider Eq. (4.11) along the punctured line $\left(t_{\text {orig }}^{1}, \ldots, t_{\text {orig }}^{N}\right) \times \mathbb{C}^{*} \subset M \times \mathbb{C}^{*}$. Let $U_{1}:=\left.\mathcal{U}\right|_{t^{\alpha}=t_{\text {orig }}^{\alpha}}$ and consider the equation

$$
\frac{\partial \xi}{\partial z}=\xi\left(U_{1}+\frac{\lambda-Q}{z}\right) \Leftrightarrow \frac{\partial \xi^{T}}{\partial z}=\left(U_{1}^{T}+\frac{\lambda-Q}{z}\right) \xi^{T},
$$

for an $N \times N$ matrix $\xi$. Then there exists a transformation $\xi^{\prime}=G(z) \xi^{T}$ with $G(z) \in$ $\operatorname{Mat}_{N, N}(\mathbb{C})[[z]], G(0)=\mathrm{Id}$, that transforms Eq. (4.12) to

$$
\frac{\partial \xi^{\prime}}{\partial z}=\left(\frac{\lambda-Q}{z}+\sum_{n \geq 1}(-1)^{n-1} R_{n}^{T} z^{n-1}\right) \xi^{\prime},
$$

where matrices $R_{n}$ satisfy $\left[Q, R_{n}\right]=n R_{n}$ (see e.g. Dubrovin 1999, Lemma 2.5). The fact that $G(z)$ and the matrices $R_{n}$ can be chosen not to depend on $\lambda$ is obvious. The matrix $\xi^{\prime}=z^{\lambda-Q_{2} \bar{R}^{T}}$, where $\bar{R}:=\sum_{n \geq 1}(-1)^{n-1} R_{n}$, satisfies equation (4.13). Therefore, the matrix $\xi=z^{\bar{R}} z^{\lambda-Q_{(}}\left(G^{T}(z)\right)^{-1}$ satisfies Eq. (4.12).

Using Eq. (4.10), we can extend the constructed function $\xi$ on the punctured line $\left(t_{\text {orig }}^{1}, \ldots, t_{\text {orig }}^{N}\right) \times \mathbb{C}^{*} \subset M \times \mathbb{C}^{*}$ to a function $\zeta$ on the whole space $M \times \mathbb{C}^{*}$. The function $\zeta$ has the form

$$
\zeta=z^{\bar{R}} z^{\lambda-Q}\left(\sum_{d \geq 0} \Omega_{0}^{d-1} z^{d}\right)
$$


where $\Omega_{0}^{d} \in \operatorname{Mat}_{N, N}(\mathcal{O}(M)), \Omega_{0}^{-1}=\operatorname{Id}$ and $\left.\left(\sum_{d \geq 0} \Omega_{0}^{d-1} z^{d}\right)\right|_{t^{\alpha}=t_{\text {orig }}^{\alpha}}=\left(G^{T}(z)\right)^{-1}$.

Since the connection $\widetilde{\nabla}^{\lambda}$ is flat, the function $\zeta$ satisfies Eq. (4.11).

Equation (4.10) for a function $\zeta$ of the form (4.14) implies that the sequence of matrices $\Omega_{0}^{d}, d \geq-1$, is a calibration of our flat F-manifold. Equation (4.11) gives

$$
\begin{aligned}
& \left(\frac{\lambda-Q}{z}+\sum_{n \geq 1}(-1)^{n-1} R_{n} z^{n-1}\right)\left(\sum_{d \geq 0} \Omega_{0}^{d-1} z^{d}\right)+\sum_{d \geq 0}(d+1) \Omega_{0}^{d} z^{d} \\
& =\left(\sum_{d \geq 0} \Omega_{0}^{d-1} z^{d}\right)\left(\mathcal{U}+\frac{\lambda-Q}{z}\right) .
\end{aligned}
$$

Taking the coefficient of $z^{d}, d \geq 0$, in this equation, we get

$$
\Omega_{0}^{d-1} \mathcal{U}=(d+1) \Omega_{0}^{d}+\left[\Omega_{0}^{d}, Q\right]+\sum_{i=1}^{d+1}(-1)^{i-1} R_{i} \Omega_{0}^{d-i}, \quad d \geq 0
$$

By (4.10), the left-hand side is equal to $E^{\theta} \frac{\partial \Omega_{0}^{d}}{\partial t^{\theta}}$. This completes the proof of the proposition.

A calibration of a conformal flat F-manifold will always be assumed to satisfy property (4.9).

From Eq. (4.9) it is easy to deduce that

$$
E^{\theta} \frac{\partial \Omega_{d}^{0}}{\partial t^{\theta}}=(d+1) \Omega_{d}^{0}+\left[\Omega_{d}^{0}, Q\right]+\sum_{i=1}^{d+1} \Omega_{d-i}^{0} R_{i}, \quad d \geq-1
$$

Remark 4.7 We see that a calibration of a conformal Frobenius manifold is the same as a calibration of the associated flat F-manifold, satisfying the additional properties $\eta \Omega_{d}^{0} \eta^{-1}=\left(\Omega_{0}^{d}\right)^{T}$ and $\eta R_{n} \eta^{-1}=(-1)^{n-1} R_{n}^{T}$.

\subsubsection{Calibrations of Extensions of Flat F-manifolds}

For an $(N+1) \times(N+1)$ matrix $A$ denote by $\pi_{N}(A)$ the $N \times N$ matrix formed by the first $N$ raws and the first $N$ columns of $A$.

Lemma 4.8 Consider a flat $F$-manifold, given by a vector potential $\left(F^{1}, \ldots, F^{N}\right)$, $F^{\alpha} \in \mathcal{O}(M)$, and its extension with a vector potential $\left(F^{1}, \ldots, F^{N+1}\right), F^{N+1} \in$ $\mathcal{O}(M \times U)$, where the open subsets $M \in \mathbb{C}^{N}$ and $U \in \mathbb{C}$ are simply connected. Suppose that the flat $F$-manifold $M \times U$ is conformal with an Euler vector field $E=\sum_{\alpha=1}^{N+1}\left(\left(1-q^{\alpha}\right) t^{\alpha}+r^{\alpha}\right) \frac{\partial}{\partial t^{\alpha}}$. Let us also fix a calibration of the flat $F$-manifold $M$, given by matrices $\Omega_{0}^{d}$ and $R_{n}$. 
Then there exists a calibration of the flat F-manifold $M \times U$ with matrices $\widetilde{\Omega}_{0}^{d}$ and $\widetilde{R}_{n}$ satisfying the properties

$$
\pi_{N}\left(\widetilde{\Omega}_{0}^{d}\right)=\Omega_{0}^{d}, \quad \pi_{N}\left(\widetilde{R}_{n}\right)=R_{n}, \quad \widetilde{\Omega}_{N+1,0}^{\leq N, d}=\left(\widetilde{R}_{n}\right)_{N+1}^{\leq N+1}=0 .
$$

Proof Consider the construction of a calibration of the conformal flat F-manifold $M \times U$ from the proof of Proposition 4.4 in more details. So we consider the differential equation

$$
\frac{\partial \widetilde{\xi}^{T}}{\partial z}=\left(\widetilde{U}_{1}^{T}+\frac{\lambda-\widetilde{Q}}{z}\right) \widetilde{\xi}^{T}
$$

for an $(N+1) \times(N+1)$ matrix $\widetilde{\xi}$, where $\left(\widetilde{U}_{1}\right)_{\beta}^{\alpha}:=\left.\left(E^{\mu} c_{\mu \beta}^{\alpha}\right)\right|_{t^{\theta}=t_{\text {orig }}^{\theta}}, 1 \leq \alpha, \beta \leq$ $N+1$, and $\widetilde{Q}:=\operatorname{diag}\left(q^{1}, \ldots, q^{N+1}\right)$. A transformation $\xi^{\prime}=\widetilde{G}(z) \widetilde{\xi}^{T}, \widetilde{G}(z)=$ Id $+\sum_{n \geq 1} \widetilde{G}_{n} z^{n}$, transforming this differential equation to the form

$$
\frac{\partial \xi^{\prime}}{\partial z}=\left(\frac{\lambda-\widetilde{Q}}{z}+\sum_{n \geq 1}(-1)^{n-1} \widetilde{R}_{n}^{T} z^{n-1}\right) \xi^{\prime}
$$

where $\left[\widetilde{Q}, \widetilde{R}_{n}\right]=n \widetilde{R}_{n}$, is determined by the recursion relation (Dubrovin 1999, equation (2.53))

$$
\begin{aligned}
& (-1)^{n-1} \widetilde{R}_{n}^{T}=\delta_{n, 1} \widetilde{U}_{1}^{T}+n \widetilde{G}_{n}+\left[\widetilde{Q}, \widetilde{G}_{n}\right] \\
& +\sum_{k=1}^{n-1}\left(\widetilde{G}_{n-k} \delta_{k, 1} \widetilde{U}_{1}^{T}-(-1)^{k-1} \widetilde{R}_{k}^{T} \widetilde{G}_{n-k}\right), \quad n \geq 1 .
\end{aligned}
$$

If one has computed the matrices $\widetilde{G}_{i}$ and $\widetilde{R}_{i}$ for $i<n$, then Eq. (4.17) determines the matrix $\widetilde{R}_{n}$ and the elements $\left(\widetilde{G}_{n}\right)_{\beta}^{\alpha}$ with $q^{\alpha}-q^{\beta} \neq-n$. The elements $\left(\widetilde{G}_{n}\right)_{\beta}^{\alpha}$ with $q^{\alpha}-q^{\beta}=-n$ can be chosen arbtitrarily. Note that $\left(\widetilde{U}_{1}\right)_{N+1}^{\leq N}=0$. Therefore, if $\left(\widetilde{R}_{i}\right)_{N+1}^{\leq N}=\left(\widetilde{G}_{i}\right)_{\leq N}^{N+1}=0$ for $i<n$, then $\left(\widetilde{R}_{n}\right)_{N+1}^{\leq N}=0$ and, choosing the elements $\left(\widetilde{G}_{n}\right)_{\alpha}^{N+1}$ with $q^{\alpha}-q^{\beta}=-n$ to be zero, we can guarantee that $\left(\widetilde{G}_{n}\right)_{\leq N}^{N+1}=0$.

Let $U_{1}:=\pi_{N}\left(\widetilde{U}_{1}\right)$ and $Q:=\pi_{N}(\widetilde{Q})$. We know that the $N \times N$ matrices $G_{n}$, given by $\left.\left(\sum_{d \geq 0} \Omega_{0}^{d-1} z^{d}\right)\right|_{t^{\alpha}=t_{\text {ori }}^{\alpha}}=\left(\operatorname{Id}+\sum_{n \geq 1} G_{n}^{T} z^{n}\right)^{-1}$, together with the matrices $R_{n}$ satisfy the equations

$$
\begin{aligned}
(-1)^{n-1} R_{n}^{T}= & \delta_{n, 1} U_{1}^{T}+n G_{n}+\left[Q, G_{n}\right] \\
& +\sum_{k=1}^{n-1}\left(G_{n-k} \delta_{k, 1} U_{1}^{T}-(-1)^{k-1} R_{k}^{T} G_{n-k}\right), \quad n \geq 1
\end{aligned}
$$


Therefore, there exist matrices $\widetilde{R}_{n}$ and $\widetilde{G}_{n}, n \geq 1$, satisfying Eq. (4.17) and the properties $\pi_{N}\left(\widetilde{R}_{n}\right)=R_{n}, \pi_{N}\left(\widetilde{G}_{n}\right)=G_{n}$ and $\left(\widetilde{\widetilde{R}}_{n}\right)_{N+1}^{\leq N}=\left(\widetilde{G}_{n}\right)_{\leq N}^{N+1}=0$. Note that the property $\left[\widetilde{Q}, \widetilde{R}_{n}\right]=n \widetilde{R}_{n}$ implies that the diagonal elements of $\widetilde{R}_{n}$ are also zero.

We construct the matrices $\widetilde{\Omega}_{0}^{d}$ as a solution of Eq. (4.6), satisfying the initial condition $\left.\left(\sum_{d \geq 0} \widetilde{\Omega}_{0}^{d-1} z^{d}\right)\right|_{t^{\alpha}=t_{\text {orig }}^{\alpha}}=\left(\mathrm{Id}+\sum_{n \geq 1} \widetilde{G}_{n}^{T} z^{n}\right)^{-1}$, and one can easily check that $\pi_{N}\left(\widetilde{\Omega}_{0}^{d}\right)=\Omega_{0}^{d}$ and $\widetilde{\Omega}_{N+1,0}^{\leq N, d}=0$.

Let us now apply this lemma to the conformal flat F-manifold, associated to a solution of the open WDVV equations, satisfying property (1.8) and the homogeneity condition (3.1). We assume that the matrices $\Omega_{0}^{d}$ and $R_{n}$ give a calibration of the Frobenius manifold. By Lemma 4.8, there exists a calibration of the flat F-manifold $M \times U$, given by matrices $\widetilde{\Omega}_{0}^{d}$ and $\widetilde{R}_{n}$, satisfying properties (4.16). Consider the matrices $\widetilde{\Omega}_{d}^{0}$ and define functions $\Phi_{\beta, d}, 1 \leq \beta \leq N, d \geq-1$, by

$$
\Phi_{\beta, d}:=\widetilde{\Omega}_{\beta, d}^{N+1,0}
$$

Lemma 4.9 1. The functions $\Phi_{\beta, d}$ together with the matrices $\widetilde{R}_{n}$ give a calibration of the function $F^{o}$. As a corollary, Lemma 3.2 is true.

2. Equation (4.18) defines a correspondence between calibrations of the flat $F$ manifold $M \times U$, satisfying properties (4.16), and calibrations of the function $F^{o}$.

Proof 1. Property (3.2) is obvious. Equations (4.8) and (4.15) for the matrices $\widetilde{\Omega}_{d}^{0}$ give exactly properties (3.3) and (3.4), respectively.

2. The fact that Eqs. (4.8) and (4.15) for the matrices $\Omega_{d}^{0}$ together with Eqs. (3.3) and (3.4) give the required equations for the matrices $\widetilde{\Omega}_{d}^{0}$ is obvious.

\subsection{Descendent Vector Potentials of a Flat F-manifold}

Consider a flat F-manifold, given by a vector potential $\left(F^{1}, \ldots, F^{N}\right), F^{\alpha} \in \mathcal{O}(M)$, and let us choose a calibration. One can immediately see that $\frac{\partial \Omega_{1,0}^{\alpha, 0}}{\partial t^{\beta}}=\delta_{\beta}^{\alpha}$, which implies that $\Omega_{1,0}^{\alpha, 0}-t^{\alpha}$ is a constant. Let us make the change of coordinates $t^{\alpha} \mapsto \Omega_{1,0}^{\alpha, 0}$, so that we have now $\Omega_{1,0}^{\alpha, 0}=t^{\alpha}$.

Let $v^{1}, \ldots, v^{N}$ be formal variables and consider the principal hierarchy associated to our flat F-manifold and its calibration (see e.g. Arsie and Lorenzoni 2018, Section $3.2)$ :

$$
\frac{\partial v^{\alpha}}{\partial t_{d}^{\beta}}=\partial_{x}\left(\left.\Omega_{\beta, d}^{\alpha, 0}\right|_{t^{\gamma}=v^{\gamma}}\right), \quad 1 \leq \alpha, \beta \leq N, \quad d \geq 0 .
$$

The flows of the principal hierarchy pairwise commute. Since $\Omega_{1,0}^{\alpha, 0}=t^{\alpha}$, we can identify $x=t_{0}^{1}$. 
Clearly the functions $v^{\alpha}=t_{0}^{\alpha}$ satisfy the subsystem of system (4.19), given by the flows $\frac{\partial}{\partial t_{0}^{\beta}}$. Denote by $\left(v^{\text {top }}\right)^{\alpha} \in \mathcal{O}(M)\left[\left[t_{\geq 1}^{*}\right]\right]$ the solution of the principal hierarchy specified by the initial condition

$$
\left.\left(v^{\text {top }}\right)^{\alpha}\right|_{t_{\geq 1}^{*}=0}=t_{0}^{\alpha}
$$

Recall that $\widetilde{t}_{d}^{\alpha}=t_{d}^{\alpha}-\delta^{\alpha, 1} \delta_{d, 1}$.

Lemma 4.10 We have

$$
\begin{gathered}
\sum_{d \geq 0} \widetilde{t}_{d+1}^{\gamma} \frac{\partial\left(v^{\text {top }}\right)^{\alpha}}{\partial t_{d}^{\gamma}}+\delta^{\alpha, 1}=0 \\
\sum_{d \geq 0} \tilde{t}_{d}^{\gamma} \frac{\partial\left(v^{\text {top }}\right)^{\alpha}}{\partial t_{d}^{\gamma}}=0
\end{gathered}
$$

Proof From the property $\frac{\partial \Omega_{d}^{0}}{\partial t^{1}}=\Omega_{d-1}^{0}, d \geq 0$, it is easy to deduce that the system

$$
\frac{\partial v^{\alpha}}{\partial \tau_{-1}}=\sum_{d \geq 0} \widetilde{t}_{d+1}^{\gamma} \frac{\partial v^{\alpha}}{\partial t_{d}^{\gamma}}+\delta^{\alpha, 1}, \quad 1 \leq \alpha \leq N
$$

is a symmetry of the principal hierarchy (4.19). Since, obviously, $\left.\frac{\partial\left(v^{\mathrm{top}}\right)^{\alpha}}{\partial \tau_{-1}}\right|_{t_{\geq 1}^{*}=0}=0$, we get Eq. (4.20).

The rescaling combined with the shift along $t_{1}^{1}$, given by

$$
\frac{\partial v^{\alpha}}{\partial \tau_{0}}=\sum_{d \geq 0} \widetilde{t}_{d}^{\gamma} \frac{\partial v^{\alpha}}{\partial t_{d}^{\gamma}}, \quad 1 \leq \alpha \leq N
$$

is also a symmetry of the principal hierarchy. We compute

$$
\left.\frac{\partial\left(v^{\text {top }}\right)^{\alpha}}{\partial \tau_{0}}\right|_{t_{\geq 1}^{*}}=t_{0}^{\alpha}-\left.\frac{\partial\left(v^{\text {top }}\right)^{\alpha}}{\partial t_{1}^{1}}\right|_{t_{\geq 1}^{*}=0}=t_{0}^{\alpha}-\frac{\partial \Omega_{1,1}^{\alpha, 0}}{\partial t_{0}^{1}}=0
$$

concluding that Eq. (4.21) is true.

Define matrices $\Omega_{q}^{p}=\left(\Omega_{\beta, q}^{\alpha, p}\right), p, q \geq 0$, by

$$
\Omega_{q}^{p}:=\sum_{i=0}^{q}(-1)^{q-i} \Omega_{0}^{p+q-i} \Omega_{i-1}^{0} \stackrel{\text { eq. }}{=}(4.7) \sum_{i=0}^{p}(-1)^{p-i} \Omega_{0}^{i-1} \Omega_{p+q-i}^{0} .
$$


We also adopt the convention $\Omega_{q}^{-1}=\Omega_{-1}^{q}:=\delta_{q, 0} \mathrm{Id}, q \geq 0$. One can easily check that

$$
\begin{aligned}
& d \Omega_{q}^{p}=\Omega_{0}^{p-1} d \Omega_{q}^{0}=d \Omega_{0}^{p} \cdot \Omega_{q-1}^{0}, \quad p, q \geq 0, \\
& \frac{\partial \Omega_{q}^{p}}{\partial t^{1}}=\Omega_{q}^{p-1}+\Omega_{q-1}^{p}, \quad p, q \geq 0, \quad p+q \geq 1 .
\end{aligned}
$$

Let

$$
\left(\Omega^{\mathrm{top}}\right)_{\beta, q}^{\alpha, p}:=\left.\Omega_{\beta, q}^{\alpha, p}\right|_{t^{\gamma} \mapsto\left(v^{\mathrm{top}}\right)^{\gamma}} .
$$

Lemma 4.11 We have $\frac{\partial\left(\Omega^{\mathrm{top}}\right)_{\beta, q}^{\alpha, p}}{\partial t_{r}^{\gamma}}=\frac{\partial\left(\Omega^{\mathrm{top}}\right)_{\gamma, r}^{\alpha, p}}{\partial t_{q}^{\beta}}, p, q, r \geq 0$.

Proof By (4.22), we have $\frac{\partial\left(\Omega^{\mathrm{top}}\right)_{\beta, q}^{\alpha, p}}{\partial t_{r}^{\gamma}}=\left(\Omega^{\mathrm{top}}\right)_{\mu, 0}^{\alpha, p-1} \frac{\partial\left(\Omega^{\mathrm{top}}\right)_{\beta, q}^{\mu, 0}}{\partial t_{r}^{\gamma}}$. The fact that the flows of the principal hierarchy pairwise commute implies that $\frac{\partial\left(\Omega^{\mathrm{top}}\right)_{\beta, q}^{\mu, 0}}{\partial t_{r}^{\gamma}}=\frac{\partial\left(\Omega^{\mathrm{top}}\right)_{\gamma, r}^{\mu, 0}}{\partial t_{q}^{\beta}}$. This completes the proof of the lemma.

We finally define the descendent vector potentials $\left(\mathcal{F}^{1, p}, \ldots, \mathcal{F}^{N, p}\right), p \geq 0$, associated to our flat F-manifold and its calibration, by

$$
\mathcal{F}^{\alpha, p}:=\sum_{q \geq 0}\left(\Omega^{\mathrm{top}}\right)_{\beta, q}^{\alpha, p} \widetilde{t}_{q}^{\beta} \in \mathcal{O}(M)\left[\left[t_{\geq 1}^{*}\right]\right], \quad p \geq 0 .
$$

Let us also adopt the convention

$$
\mathcal{F}^{\alpha, p}:=(-1)^{p+1} \widetilde{t}_{-p-1}^{\alpha}, \quad \text { if } p<0
$$

Proposition 4.12 1. We have

$$
\begin{gathered}
\frac{\partial \mathcal{F}^{\alpha, p}}{\partial t_{q}^{\beta}}=\left(\Omega^{\text {top }}\right)_{\beta, q}^{\alpha, p}, \quad p \geq 0, \\
\sum_{q \geq 0} \widetilde{t}_{q+1}^{\beta} \frac{\partial \mathcal{F}^{\alpha, p}}{\partial t_{q}^{\beta}}+\mathcal{F}^{\alpha, p-1}=0, \quad p \in \mathbb{Z} .
\end{gathered}
$$

2. The difference $\left.\mathcal{F}^{\alpha, 0}\right|_{t_{\geq 1}^{*}=0}-F^{\alpha}$ is at most linear in the variables $t^{1}, \ldots, t^{N}$.

Proof 1. We compute

$$
\begin{aligned}
\frac{\partial \mathcal{F}^{\alpha, p}}{\partial t_{q}^{\beta}}= & \sum_{r \geq 0} \frac{\partial\left(\Omega^{\mathrm{top}}\right)_{\gamma, r}^{\alpha, p}}{\partial t_{q}^{\beta}} \widetilde{t}_{r}^{\gamma}+\left(\Omega^{\mathrm{top}}\right)_{\beta, q}^{\alpha, p} \stackrel{\text { Lemma }}{=}{ }^{4.11} \sum_{r \geq 0} \frac{\partial\left(\Omega^{\mathrm{top}}\right)_{\beta, q}^{\alpha, p}}{\partial t_{r}^{\gamma}} \widetilde{t}_{r}^{\gamma} \\
& +\left(\Omega^{\mathrm{top}}\right)_{\beta, q}^{\alpha, p} \stackrel{\text { eq. }}{=} \stackrel{(4.21)}{=}\left(\Omega^{\mathrm{top}}\right)_{\beta, q}^{\alpha, p} .
\end{aligned}
$$


For $p<0$ Eq. (4.25) is obvious. Suppose $p \geq 0$, then we have

$$
\begin{aligned}
\sum_{q \geq 0} \widetilde{t}_{q+1}^{\beta} \frac{\partial \mathcal{F}^{\alpha, p}}{\partial t_{q}^{\beta}} & =\sum_{q, r \geq 0} \frac{\partial\left(\Omega^{\text {top }}\right)_{\gamma, r}^{\alpha, p}}{\partial t_{q}^{\beta}} \widetilde{t}_{q+1}^{\beta} \widetilde{t}_{r}^{\gamma}+\sum_{q \geq 0} \widetilde{t}_{q+1}^{\beta}\left(\Omega^{\text {top }}\right)_{\beta, q}^{\alpha, p} \stackrel{\text { eq. }}{=}{ }^{(4.20)} \\
& =-\sum_{r \geq 0} \frac{\partial\left(\Omega^{\text {top }}\right)_{\gamma, r}^{\alpha, p}}{\partial\left(v^{\text {top }}\right)^{1}} \widetilde{t}_{r}^{\gamma}+\sum_{q \geq 0} \widetilde{t}_{q+1}^{\beta}\left(\Omega^{\text {top }}\right)_{\beta, q}^{\alpha, p} \stackrel{\text { eq. }}{=} \stackrel{(4.23)}{=} \\
& =-\sum_{r \geq 0}\left(\Omega^{\text {top }}\right)_{\gamma, r}^{\alpha, p} \widetilde{t}_{r+1}^{\gamma}-\sum_{r \geq 0}\left(\Omega^{\text {top }}\right)_{\gamma, r}^{\alpha, p-1} \widetilde{t}_{r}^{\gamma}+\sum_{q \geq 0} \widetilde{t}_{q+1}^{\beta}\left(\Omega^{\text {top }}\right)_{\beta, q}^{\alpha, p} \\
& =-\mathcal{F}^{\alpha, p-1} .
\end{aligned}
$$

2. By the first part of the proposition, $\frac{\partial}{\partial t_{0}^{\beta}}\left(\left.\mathcal{F}^{\alpha, 0}\right|_{t_{\geq 1}^{*}=0}\right)=\Omega_{\beta, 0}^{\alpha, 0}$. Since $\frac{\partial \Omega_{\beta, 0}^{\alpha, 0}}{\partial t^{\gamma}}=c_{\beta \gamma}^{\alpha}$, the second part of the proposition is also proved.

Remark 4.13 Consider a Frobenius manifold, its calibration and the associated flat F-manifold. Then the functions $\mathcal{F}^{\alpha, p}$ are related to the descendent potential $\mathcal{F}$ of the Frobenius manifold by $\mathcal{F}^{\alpha, p}=\eta^{\alpha \mu} \frac{\partial \mathcal{F}}{\partial t_{p}^{\mu}}$.

Consider a conformal Frobenius manifold together with a calibration and a solution $F^{o}$ of the open WDVV equations, satisfying properties (1.8), (3.1), also with a calibration. We have the associated flat F-manifold with the vector potential $\left(\eta^{1 \mu} \frac{\partial F}{\partial t^{\mu}}, \ldots, \eta^{N \mu} \frac{\partial F}{\partial t^{\mu}}, F^{o}\right)$. Immediately from the definitions and Lemma 4.9 we see that if $\left(\mathcal{F}^{1, p}, \ldots, \mathcal{F}^{N+1, p}\right), p \geq 0$, are the decendent vector potentials of this flat F-manifold, then $\mathcal{F}^{\alpha, p}=\eta^{\alpha \mu} \frac{\partial \mathcal{F}}{\partial t_{p}^{\mu}}, 1 \leq \alpha \leq N$, and $\mathcal{F}^{N+1,0}=\mathcal{F}^{o}$. Therefore, Lemma 3.3 follows from Proposition 4.12 and Eq. (4.22).

\subsection{Virasoro Constraints}

We consider a conformal flat F-manifold, given by a vector potential $\left(F^{1}, \ldots, F^{N}\right)$, $F^{\alpha} \in \mathcal{O}(M)$, and an Euler vector field (2.1), its calibration, described by matrices $\Omega_{0}^{d}, d \geq-1$, and $R_{n}, n \geq 1$, and the associated descendent vector potentials $\left(\mathcal{F}^{1, p}, \ldots, \mathcal{F}^{N, p}\right), p \geq 0$.

Recall that $R=\sum_{i \geq 1} R_{i}$. Let $\lambda$ be a complex parameter and define

$$
\mu^{\alpha}:=q^{\alpha}+\lambda-\frac{3}{2}, \quad \mu:=\operatorname{diag}\left(\mu^{1}, \ldots, \mu^{N}\right)
$$

Define the following expressions, depending on the parameter $\lambda$ :

$$
\begin{aligned}
A_{m}^{\alpha}:= & \sum_{\substack{d_{1} \geq-1, d_{2} \in \mathbb{Z}\\
}}(-1)^{d_{2}+1}\left(\left[P_{m}\left(\mu-d_{2}, R\right)\right]_{m-1-d_{1}-d_{2}}\right)_{\mu}^{\gamma}\left(\Omega^{\mathrm{top}}\right)_{\gamma, d_{1}}^{\alpha, 0} \mathcal{F}^{\mu, d_{2}}, \\
& 1 \leq \alpha \leq N, \quad m \geq-1 .
\end{aligned}
$$


Proposition 4.14 We have $A_{m}^{\alpha}=0,1 \leq \alpha \leq N, m \geq-1$.

Remark 4.15 For a Frobenius manifold the expressions $A_{m}^{\alpha}$ have the following interpretation:

$$
\left.A_{m}^{\alpha}\right|_{\lambda=\frac{3-\delta}{2}}=\eta^{\alpha \mu} \frac{\partial}{\partial t_{0}^{\mu}}\left[\operatorname{Coef}_{\varepsilon^{-2}}\left(\frac{L_{m} e^{\varepsilon^{-2} \mathcal{F}}}{e^{\varepsilon^{-2} \mathcal{F}}}\right)\right] \text {, }
$$

where $L_{m}$ are the Virasoro operators described in Sect. 2.2. Therefore, we consider Proposition 4.14 as a generalization of the Virasoro constraints (1.4) for an arbitrary conformal flat F-manifold.

Proof of Proposition 4.14 During the proof of the proposition, for the sake of shortness, we will denote the functions $\left(v^{\text {top }}\right)^{\alpha}$ and $\left(\Omega^{\text {top }}\right)_{\beta, q}^{\alpha, p}$ by $v^{\alpha}$ and $\Omega_{\beta, q}^{\alpha, p}$, respectively. We will also denote the function $\left.c_{\beta \gamma}^{\alpha}\right|_{t^{\theta} \mapsto\left(v^{\text {top }}\right)^{\theta}}$ by $c_{\beta \gamma}^{\alpha}$ and the function $\left(1-q^{\alpha}\right)\left(v^{\text {top }}\right)^{\alpha}+r^{\alpha}$ by $E^{\alpha}$.

Define an operator $\mathcal{B}=\left(\mathcal{B}_{\beta}^{\alpha}\right)$, depending on the parameter $\lambda$, by

$$
\mathcal{B}_{\beta}^{\alpha}:=E^{\mu} c_{\mu \beta}^{\alpha} \partial_{x}+v_{x}^{\gamma}\left(\lambda-1+q^{\beta}\right) c_{\gamma \beta}^{\alpha}=E^{\mu} c_{\mu \beta}^{\alpha} \partial_{x}+v_{x}^{\gamma}\left(\mu^{\beta}+\frac{1}{2}\right) c_{\gamma \beta}^{\alpha} .
$$

Remark 4.16 For a Frobenius manifold the operator $\left.\mathcal{B}\right|_{\lambda=\frac{3-\delta}{2}} \partial_{x}^{-1}$ coincides with the recursion operator $\mathcal{R}$ from the paper (Dubrovin and Zhang 1999, equation (3.36)).

We begin with the following lemma.

Lemma 4.17 We have $\mathcal{B} \Omega_{d}^{0}=\partial_{x}\left(\Omega_{d+1}^{0}\left(\mu+d+\frac{3}{2}\right)+\sum_{i=1}^{d+2} \Omega_{d+1-i}^{0} R_{i}\right), d \geq-1$.

Proof Note that $\mathcal{B}_{\beta}^{\alpha}=\partial_{x} \cdot\left(E^{\mu} c_{\mu \beta}^{\alpha}\right)+v_{x}^{\gamma}\left(\lambda+q^{\alpha}-2\right) c_{\gamma \beta}^{\alpha}$. This follows from the property $E^{\mu} \frac{\partial c_{\beta \gamma}^{\alpha}}{\partial v^{\mu}}=\left(q^{\beta}+q^{\gamma}-q^{\alpha}\right) c_{\beta \gamma}^{\alpha}$ which is equivalent to the homogeneity condtition (4.4). We then compute $\mathcal{B} \Omega_{d}^{0}=\partial_{x}\left(E^{\mu} \frac{\partial \Omega_{d+1}^{0}}{\partial v^{\mu}}+(\lambda+Q-2) \Omega_{d+1}^{0}\right)$, that, by Eq. (4.15), implies the lemma.

Denote by $A_{m}$ the column vector $\left(A_{m}^{1}, \ldots, A_{m}^{N}\right)$.

Lemma 4.18 We have

$$
\begin{aligned}
& \mathcal{B} A_{m}-\partial_{x} A_{m+1} \\
& =\left(\frac{1}{2}-\mu\right) \sum_{d_{1}, d_{2} \geq-1}(-1)^{d_{2}+1} \Omega_{d_{1}}^{0}\left[P_{m}\left(\mu-d_{2}, R\right)\right]_{m-d_{1}-d_{2}} \Omega_{0}^{d_{2}} \cdot e, \quad m \geq-1,
\end{aligned}
$$

where e denotes the unit vector $(1,0, \ldots, 0)$. 
Proof We compute

$$
\begin{aligned}
& \mathcal{B}_{\mu}^{\alpha} A_{m}^{\mu}=\sum_{d_{1} \geq-1, d_{2} \in \mathbb{Z}}(-1)^{d_{2}+1}\left(\left[P_{m}\left(\mu-d_{2}, R\right)\right]_{m-1-d_{1}-d_{2}}\right)_{\nu}^{\gamma} \mathcal{B}_{\mu}^{\alpha} \Omega_{\gamma, d_{1}}^{\mu, 0} \cdot \mathcal{F}^{\nu, d_{2}} \\
& +\sum_{d \geq-1}\left(\left[P_{m}(\mu+1, R)\right]_{m-d}\right)_{1}^{\gamma} E^{\theta} \frac{\partial \Omega_{\gamma, d+1}^{\alpha, 0}}{\partial v^{\theta}} \\
& +\sum_{d_{1} \geq-1, d_{2} \geq 0}(-1)^{d_{2}+1}\left(\left[P_{m}\left(\mu-d_{2}, R\right)\right]_{m-1-d_{1}-d_{2}}\right)_{\nu}^{\gamma} E^{\theta} \frac{\partial \Omega_{\gamma, d_{1}+1}^{\alpha, 0}}{\partial v^{\theta}} \Omega_{1,0}^{\nu, d_{2}} \\
& =\sum_{d_{1} \geq-1, d_{2} \in \mathbb{Z}}(-1)^{d_{2}+1}\left(\left[P_{m}\left(\mu-d_{2}, R\right)\right]_{m-1-d_{1}-d_{2}}\right)_{\nu}^{\gamma} \mathcal{B}_{\mu}^{\alpha} \Omega_{\gamma, d_{1}}^{\mu, 0} \cdot \mathcal{F}^{\nu, d_{2}} \\
& +\sum_{d_{1}, d_{2} \geq-1}(-1)^{d_{2}+1}\left(\left[P_{m}\left(\mu-d_{2}, R\right)\right]_{m-1-d_{1}-d_{2}}\right)_{v}^{\gamma} E^{\theta} \frac{\partial \Omega_{\gamma, d_{1}+1}^{\alpha, 0}}{\partial v^{\theta}} \Omega_{1,0}^{v, d_{2}} .
\end{aligned}
$$

On the other hand, we have

$$
\begin{aligned}
\partial_{x} A_{m+1}^{\alpha}= & \sum_{d_{1} \geq 0, d_{2} \in \mathbb{Z}}(-1)^{d_{2}+1}\left(\left[P_{m+1}\left(\mu-d_{2}, R\right)\right]_{m-d_{1}-d_{2}}\right)_{\mu}^{\gamma} \partial_{x} \Omega_{\gamma, d_{1}}^{\alpha, 0} \cdot \mathcal{F}^{\mu, d_{2}} \\
& +\sum_{d_{1}, d_{2} \geq-1}(-1)^{d_{2}+1}\left(\left[P_{m+1}\left(\mu-d_{2}, R\right)\right]_{m-d_{1}-d_{2}}\right)_{\mu}^{\gamma} \Omega_{\gamma, d_{1}}^{\alpha, 0} \Omega_{1,0}^{\mu, d_{2}}
\end{aligned}
$$

Let us show that the expression in line (4.26) is equal to the expression in line (4.28). Using Lemma 4.17, we rewrite the first one as follows:

$$
\begin{aligned}
& \sum_{d_{1} \geq-1, d_{2} \in \mathbb{Z}}(-1)^{d_{2}+1} \mathcal{F}^{v, d_{2}}\left(\left[P_{m}\left(\mu-d_{2}, R\right)\right]_{m-1-d_{1}-d_{2}}\right)_{\nu}^{\gamma} \partial_{x}\left(\left(\mu^{\gamma}+d_{1}+\frac{3}{2}\right) \Omega_{\gamma, d_{1}+1}^{\alpha, 0}\right. \\
& \left.+\sum_{i=1}^{d_{1}+1} \Omega_{\theta, d_{1}+1-i}^{\alpha, 0}\left(R_{i}\right)_{\gamma}^{\theta}\right) \\
= & \sum_{d_{1} \geq-1, d_{2} \in \mathbb{Z}}(-1)^{d_{2}+1} \mathcal{F}^{v, d_{2}}\left(\left[P_{m}\left(\mu-d_{2}, R\right)\left(\mu-d_{2}+m+\frac{1}{2}\right)\right]_{m-1-d_{1}-d_{2}}\right)_{\nu}^{\gamma} \partial_{x} \Omega_{\gamma, d_{1}+1}^{\alpha, 0} \\
& +\sum_{d_{1} \geq-1, d_{2} \in \mathbb{Z}} \sum_{i=1}^{d_{1}+1}(-1)^{d_{2}+1} \mathcal{F}^{v, d_{2}}\left(\left[R_{i} P_{m}\left(\mu-d_{2}, R\right)\right]_{m-1-d_{1}-d_{2}+i}\right)_{\nu}^{\theta} \partial_{x} \Omega_{\theta, d_{1}+1-i}^{\alpha, 0} \\
= & \sum_{d_{1} \geq-1, d_{2} \in \mathbb{Z}}(-1)^{d_{2}+1} \mathcal{F}^{v, d_{2}}\left(\left[P_{m}\left(\mu-d_{2}, R\right)\left(\mu-d_{2}+m+\frac{1}{2}\right)+\right.\right. \\
& \left.\left.+R P_{m}\left(\mu-d_{2}, R\right)\right]_{m-1-d_{2}-d_{2}}\right)_{\nu}^{\gamma} \partial_{x} \Omega_{\gamma, d_{1}+1}^{\alpha, 0}
\end{aligned}
$$




$$
=\sum_{d_{1} \geq-1, d_{2} \in \mathbb{Z}}(-1)^{d_{2}+1} \mathcal{F}^{\nu, d_{2}}\left(\left[P_{m+1}\left(\mu-d_{2}, R\right)\right]_{m-1-d_{1}-d_{2}}\right)_{\nu}^{\gamma} \partial_{x} \Omega_{\gamma, d_{1}+1}^{\alpha, 0},
$$

and we see that the last expression coincides with the expression in line (4.28).

Using Eq. (4.15), we transform the expression in line (4.27):

$$
\begin{aligned}
& \sum_{d_{1} \geq 0, d_{2} \geq-1}(-1)^{d_{2}+1}\left(\left[P_{m}\left(\mu-d_{2}, R\right)\right]_{m-d_{1}-d_{2}}\right)_{\nu}^{\gamma}\left(\left(\mu^{\gamma}-\mu^{\alpha}+d_{1}+1\right) \Omega_{\gamma, d_{1}}^{\alpha, 0}\right. \\
& \left.+\sum_{i=1}^{d_{1}+1} \Omega_{\theta, d_{1}-i}^{\alpha, 0}\left(R_{i}\right)_{\gamma}^{\theta}\right) \Omega_{1,0}^{\nu, d_{2}} \\
= & \sum_{d_{1} \geq 0, d_{2} \geq-1}(-1)^{d_{2}+1}\left(\left[P_{m}\left(\mu-d_{2}, R\right)\left(\mu-\mu^{\alpha}+m-d_{2}+1\right)\right]_{m-d_{1}-d_{2}}\right)_{v}^{\gamma} \Omega_{\gamma, d_{1}}^{\alpha, 0} \Omega_{1,0}^{\nu, d_{2}} \\
& +\sum_{d_{1} \geq 0, d_{2} \geq-1} \sum_{i=1}^{d_{1}+1}(-1)^{d_{2}+1}\left(\left[R_{i} P_{m}\left(\mu-d_{2}, R\right)\right]_{m-d_{1}-d_{2}+i}\right)_{v}^{\gamma} \Omega_{\gamma, d_{1}-i}^{\alpha, 0} \Omega_{1,0}^{\nu, d_{2}} \\
= & \left(\frac{1}{2}-\mu^{\alpha}\right) \sum_{d_{1} \geq 0, d_{2} \geq-1}(-1)^{d_{2}+1}\left(\left[P_{m}\left(\mu-d_{2}, R\right)\right]_{m-d_{1}-d_{2}}\right)_{\nu}^{\gamma} \Omega_{\gamma, d_{1}}^{\alpha, 0} \Omega_{1,0}^{\nu, d_{2}} \\
& +\sum_{d_{1} \geq 0, d_{2} \geq-1}(-1)^{d_{2}+1}\left(\left[P_{m}\left(\mu-d_{2}, R\right)\left(\mu+m-d_{2}+\frac{1}{2}\right)\right]_{m-d_{1}-d_{2}}\right)_{v}^{\gamma} \Omega_{\gamma, d_{1}}^{\alpha, 0} \Omega_{1,0}^{\nu, d_{2}} \\
& +\sum_{d_{1}, d_{2} \geq-1}(-1)^{d_{2}+1}\left(\left[R P_{m}\left(\mu-d_{2}, R\right)\right]_{m-d_{1}-d_{2}}\right)_{\nu}^{\gamma} \Omega_{\gamma, d_{1}}^{\alpha, 0} \Omega_{1,0}^{\nu, d_{2}} .
\end{aligned}
$$

On the other hand, the expression in line (4.29) is equal to

$$
\begin{aligned}
& \sum_{d_{1}, d_{2} \geq-1}(-1)^{d_{2}+1}\left(\left[P_{m}\left(\mu-d_{2}, R\right)\left(\mu+m-d_{2}+\frac{1}{2}\right)\right]_{m-d_{1}-d_{2}}\right)_{\nu}^{\gamma} \Omega_{\gamma, d_{1}}^{\alpha, 0} \Omega_{1,0}^{\nu, d_{2}} \\
& \quad+\sum_{d_{1}, d_{2} \geq-1}(-1)^{d_{2}+1}\left(\left[R P_{m}\left(\mu-d_{2}, R\right)\right]_{m-d_{1}-d_{2}}\right)_{\nu}^{\gamma} \Omega_{\gamma, d_{1}}^{\alpha, 0} \Omega_{1,0}^{\nu, d_{2}}
\end{aligned}
$$

As a result, we get

$$
\begin{aligned}
\mathcal{B}_{\mu}^{\alpha} A_{m}^{\mu}-\partial_{x} A_{m+1}^{\alpha} & \left(\frac{1}{2}-\mu^{\alpha}\right) \sum_{d_{1} \geq 0, d_{2} \geq-1}(-1)^{d_{2}+1}\left(\left[P_{m}\left(\mu-d_{2}, R\right)\right]_{m-d_{1}-d_{2}}\right)_{\nu}^{\gamma} \Omega_{\gamma, d_{1}}^{\alpha, 0} \Omega_{1,0}^{\nu, d_{2}} \\
= & -\sum_{d_{2} \geq-1}(-1)^{d_{2}+1}\left(\left[P_{m}\left(\mu-d_{2}, R\right)\left(\mu+m-d_{2}+\frac{1}{2}\right)\right]_{m+1-d_{2}}\right)_{v}^{\alpha} \Omega_{1,0}^{v, d_{2}} \\
= & \left(\frac{1}{2}-\mu^{\alpha}\right) \sum_{d_{1}, d_{2} \geq-1}(-1)^{d_{2}+1}\left(\left[P_{m}\left(\mu-d_{2}, R\right)\right]_{m-d_{1}-d_{2}}\right)_{\nu}^{\gamma} \Omega_{\gamma, d_{1}}^{\alpha, 0} \Omega_{1,0}^{\nu, d_{2}},
\end{aligned}
$$

as required. 
For $m \geq n \geq-1$ denote

$$
C_{m, n}:=\sum_{d_{1}, d_{2} \geq-1}(-1)^{d_{2}+1} \Omega_{d_{1}}^{0}\left[P_{n}\left(\mu-d_{2}, R\right)\right]_{m-d_{1}-d_{2}} \Omega_{0}^{d_{2}} .
$$

Lemma 4.19 We have $C_{m, n}=0, m \geq n \geq-1$.

Proof We proceed by induction on $n$. By Eq. (4.7), we have

$$
C_{m,-1}=\sum_{\substack{d_{1}, d_{2} \geq-1 \\ d_{1}+d_{2}=m}}(-1)^{d_{2}+1} \Omega_{d_{1}}^{0} \Omega_{0}^{d_{2}}=0, \quad m \geq-1 .
$$

Suppose that $m \geq n \geq 0$. We compute

$$
\begin{aligned}
C_{m, n}= & \sum_{d_{1}, d_{2} \geq-1}(-1)^{d_{2}+1} \Omega_{d_{1}}^{0}\left[P_{n-1}\left(\mu-d_{2}, R\right)\left(\mu-d_{2}+n-\frac{1}{2}\right)\right. \\
& \left.+R P_{n-1}\left(\mu-d_{2}, R\right)\right]_{m-d_{1}-d_{2}} \Omega_{0}^{d_{2}} \\
= & \sum_{d_{1}, d_{2} \geq-1}(-1)^{d_{2}+1} \Omega_{d_{1}}^{0}\left[\left(\mu-m+n+d_{1}-\frac{1}{2}\right) P_{n-1}\left(\mu-d_{2}, R\right)\right. \\
& \left.+R P_{n-1}\left(\mu-d_{2}, R\right)\right]_{m-d_{1}-d_{2}} \Omega_{0}^{d_{2}} .
\end{aligned}
$$

Using property (4.15), we transform the last expression in the following way:

$$
\begin{aligned}
& \left(\mu-m+n-\frac{3}{2}\right) C_{m, n-1}+\sum_{d_{1}, d_{2} \geq-1}(-1)^{d_{2}+1} E^{\theta} \frac{\partial \Omega_{d_{1}}^{0}}{\partial v^{\theta}}\left[P_{n-1}\left(\mu-d_{2}, R\right)\right]_{m-d_{1}-d_{2}} \Omega_{0}^{d_{2}} \\
& -\sum_{d_{1}, d_{2} \geq-1} \sum_{i=1}^{d_{1}+1}(-1)^{d_{2}+1} \Omega_{d_{1}-i}^{0}\left[R_{i} P_{n-1}\left(\mu-d_{2}, R\right)\right]_{m-d_{1}-d_{2}+i} \Omega_{0}^{d_{2}} \\
& +\sum_{d_{1}, d_{2} \geq-1}(-1)^{d_{2}+1} \Omega_{d_{1}}^{0}\left[R P_{n-1}\left(\mu-d_{2}, R\right)\right]_{m-d_{1}-d_{2}} \Omega_{0}^{d_{2}} .
\end{aligned}
$$

One can see that the last two expressions cancel each other and, as a result,

$$
C_{m, n}=\left(\mu-m+n-\frac{3}{2}\right) C_{m, n-1}+E^{\theta} \frac{\partial \Omega_{0}^{0}}{\partial v^{\theta}} C_{m-1, n-1}
$$

that, by the induction assumption, is equal to zero. The lemma is proved.

Lemmas 4.18 and 4.19 imply that $\mathcal{B} A_{m}=\partial_{x} A_{m+1}$ for $m \geq-1$. Introduce a differential operator $L:=\sum_{d \geq 0} \widetilde{t}_{d+1}^{\gamma} \frac{\partial}{\partial t_{d}^{\gamma}}$. 
Lemma 4.20 We have $L A_{m}^{\alpha}=(-m-1) A_{m-1}^{\alpha}, m \geq 0$.

Proof Using formulas (4.20), (4.25) and the formula $\frac{\partial \Omega_{d}^{0}}{\partial v^{1}}=\Omega_{d-1}^{0}, d \geq 0$, we compute

$$
\begin{aligned}
L A_{m}^{\alpha}= & -\sum_{d_{1} \geq 0, d_{2} \in \mathbb{Z}}(-1)^{d_{2}+1}\left(\left[P_{m}\left(\mu-d_{2}, R\right)\right]_{m-1-d_{1}-d_{2}}\right)_{\mu}^{\gamma} \Omega_{\gamma, d_{1}-1}^{\alpha, 0} \mathcal{F}^{\mu, d_{2}} \\
& -\sum_{d_{1} \geq-1, d_{2} \in \mathbb{Z}}(-1)^{d_{2}+1}\left(\left[P_{m}\left(\mu-d_{2}, R\right)\right]_{m-1-d_{1}-d_{2}}\right)_{\mu}^{\gamma} \Omega_{\gamma, d_{1}}^{\alpha, 0} \mathcal{F}^{\mu, d_{2}-1} \\
= & \sum_{d_{1} \geq-1, d_{2} \in \mathbb{Z}}(-1)^{d_{2}+1}\left(\left[P_{m}\left(\mu-d_{2}-1, R\right)-P_{m}\left(\mu-d_{2}, R\right)\right]_{m-2-d_{1}-d_{2}}\right)_{\mu}^{\gamma} \Omega_{\gamma, d_{1}}^{\alpha, 0} \mathcal{F}^{\mu, d_{2}} .
\end{aligned}
$$

Since $P_{m}\left(\mu-d_{2}-1, R\right)-P_{m}\left(\mu-d_{2}, R\right)=(-m-1) P_{m-1}\left(\mu-d_{2}, R\right)$, the lemma is proved.

Lemma 4.21 We have $\left.A_{m}^{\alpha}\right|_{t_{>1}^{*}=0}=0, m \geq-1$.

Proof During the proof of this lemma we return to the initial notation, where $\Omega_{\beta, q}^{\alpha, p}$ is a function of $t^{1}, \ldots, t^{N}$. Note that, by Eqs. (4.24) and (4.25), we have $\left.\mathcal{F}^{\alpha, p}\right|_{t_{\geq 1}^{*}=0}=$ $\Omega_{1,0}^{\alpha, p+1}, p \geq 0$. Therefore, we have

$$
\begin{aligned}
\left.A_{m}^{\alpha}\right|_{t_{\geq 1}^{*}=0}= & \sum_{d \geq-1}\left(\left[P_{m}(\mu+1, R)\right]_{m-d}\right)_{\mu}^{\gamma} t_{0}^{\mu} \Omega_{\gamma, d}^{\alpha, 0} \\
& -\sum_{d \geq-1}\left(\left[P_{m}(\mu+2, R)\right]_{m+1-d}\right)_{1}^{\gamma} \Omega_{\gamma, d}^{\alpha, 0} \\
& +\sum_{d_{1} \geq-1, d_{2} \geq 0}(-1)^{d_{2}+1}\left(\left[P_{m}\left(\mu-d_{2}, R\right)\right]_{m-1-d_{1}-d_{2}}\right)_{\mu}^{\gamma} \Omega_{\gamma, d_{1}}^{\alpha, 0} \Omega_{1,0}^{\mu, d_{2}+1} \\
= & -\sum_{d_{1}, d_{2} \geq-1}(-1)^{d_{2}+1}\left(\left[P_{m}\left(\mu+1-d_{2}, R\right)\right]_{m-d_{1}-d_{2}}\right)_{\mu}^{\gamma} \Omega_{\gamma, d_{1}}^{\alpha, 0} \Omega_{1,0}^{\mu, d_{2}} \\
= & -\left.\left(C_{m, m}\right)_{1}^{\alpha}\right|_{\substack{t_{\geq 1}^{*}=0 \\
\lambda \mapsto \lambda+1}},
\end{aligned}
$$

which, by Lemma 4.19, is equal to zero.

Let us now prove that $A_{m}^{\alpha}=0$ by induction on $m$. We have

$$
A_{-1}^{\alpha}=\sum_{\substack{d \geq-1, d_{2} \in \mathbb{Z} \\ d_{1}+d_{2}=-2}}(-1)^{d_{2}+1} \Omega_{\gamma, d_{1}}^{\alpha, 0} \mathcal{F}^{\gamma, d_{2}}=t_{0}^{\alpha}+\sum_{d \geq 0} \Omega_{\gamma, d}^{\alpha, 0} \widetilde{t}_{d+1}^{\alpha}=t_{0}^{\alpha}+L \mathcal{F}^{\alpha, 0} \stackrel{\text { eq.(4.25) }}{=} 0
$$

Suppose that $m \geq 0$. Let us express $A_{m}^{\alpha}$ as a power series in the variables $\widehat{t}_{d}^{\beta}$ defined by

$$
\widehat{t}_{d}^{\beta}:= \begin{cases}t_{0}^{\beta}-t_{\text {orig }}^{\beta}, & \text { if } d=0 \\ t_{d}^{\beta}, & \text { if } d \geq 1\end{cases}
$$


From the induction assumption, Lemma 4.20 and the fact that $\mathcal{B} A_{m-1}=\partial_{x} A_{m}$ it follows that

$$
\sum_{d \geq 0} \widehat{t}_{d+1}^{\gamma} \frac{\partial A_{m}^{\alpha}}{\partial \widehat{t}_{d}^{\gamma}}=0
$$

We also know that $\left.A_{m}^{\alpha}\right|_{\hat{t}_{*}^{*}=0}=0$. We see that the proof of the proposition is completed by the following lemma.

Lemma 4.22 Let $f$ be a formal power series in the variables $\widehat{t}_{d}^{\alpha}$ such that $\sum_{d \geq 0} \widehat{t}_{d+1}^{\alpha} \frac{\partial f}{\partial \widehat{t}_{d}^{\alpha}}=0$ and $\left.f\right|_{\hat{t}_{*}^{*}=0}=0$. Then $f=0$.

Proof Denote by $\mathcal{P}$ the set of all finite sequences $\lambda=\left(\lambda_{1}, \ldots, \lambda_{l}\right), l \geq 0, \lambda_{i} \in \mathbb{Z}$, such that $\lambda_{1} \geq \cdots \geq \lambda_{l} \geq 0$. We also denote $l(\lambda):=l$ and $|\lambda|:=\sum \lambda_{i}$. Then we can express the power series $f$ in the following way:

$$
f=\sum_{\lambda^{1}, \ldots, \lambda^{N} \in \mathcal{P}} a_{\lambda^{1}, \ldots, \lambda^{N}} \widehat{t}_{\lambda^{1}}^{1} \cdots \widehat{t}_{\lambda^{N}}^{N}, \quad a_{\lambda^{1}, \ldots, \lambda^{N}} \in \mathbb{C},
$$

where for an element $\lambda=\left(\lambda_{1}, \ldots, \lambda_{l}\right) \in \mathcal{P}$ we denote $\widehat{t}_{\lambda}^{\alpha}:=\widehat{t}_{\lambda_{1}}^{\alpha} \cdots \widehat{t}_{\lambda_{l}}^{\alpha}$. Note that if we assign to a monomial $\widehat{t}_{\lambda^{1}}^{1} \cdots \widehat{t}_{\lambda^{N}}^{N}$ degree $\sum\left|\lambda^{i}\right|$, then the operator $\sum_{d \geq 0} \widehat{t}_{d+1}^{\alpha} \frac{\partial}{\partial t_{d}^{\alpha}}$ becomes homogeneous of degree 1 . Denote by $V_{d}$ the subspace of $\mathbb{C}\left[\left[\widehat{t}_{*}^{*}\right]\right]$ spanned by monomials of degree $d$. Using the lexicographical order on monomials $\widehat{t}_{\lambda^{1}}^{1} \cdots \widehat{t}_{\lambda^{N}}^{N}$, it is easy to check that the map $\sum_{k \geq 0} \widehat{t}_{k+1}^{\alpha} \frac{\partial}{\partial \widehat{t}_{k}^{\alpha}}: V_{d} \rightarrow V_{d+1}$ is injective for $d \geq 1$; and for $d=0$ its kernel consists of constants. This completes the proof of the lemma.

\subsection{Proof of Theorem 3.4}

Let us apply Proposition 4.14 to the flat F-manifold associated to the function $F^{o}$. We choose $\lambda=\frac{3-\delta}{2}$, then we have

$$
A_{m}^{N+1}=\sum_{d_{1} \geq-1, d_{2} \in \mathbb{Z}}(-1)^{d_{2}+1}\left(\left[P_{m}\left(\tilde{\mu}-d_{2}, \widetilde{R}\right)\right]_{m-1-d_{1}-d_{2}}\right)_{\mu}^{\gamma} \Phi_{\gamma, d_{1}}^{\text {top }} \mathcal{F}^{\mu, d_{2}}
$$

Since $\widetilde{R}_{N+1}^{\alpha}=0$, we have $\left(\left[P_{m}\left(\tilde{\mu}-d_{2}, \widetilde{R}\right)\right]_{m-1-d_{1}-d_{2}}\right)_{N+1}^{\gamma}=0$ for $1 \leq \gamma \leq N$. Therefore,

$$
\begin{aligned}
A_{m}^{N+1}= & \sum_{d_{1} \geq 0, d_{2} \in \mathbb{Z}} \sum_{1 \leq \gamma, \mu \leq N}(-1)^{d_{2}+1}\left(\left[P_{m}\left(\tilde{\mu}-d_{2}, \widetilde{R}\right)\right]_{m-1-d_{1}-d_{2}}\right)_{\mu}^{\gamma} \frac{\partial \mathcal{F}^{o}}{\partial t_{d_{1}}^{\gamma}} \mathcal{F}^{\mu, d_{2}} \\
& +\sum_{d \geq 0}\left(\left[P_{m}(\tilde{\mu}+d+1, \widetilde{R})\right]_{m+d+1}\right)_{\alpha}^{N+1} \tilde{t}_{d}^{\alpha} \\
& +\sum_{d, k \geq 0}\left(\left[P_{m}(\tilde{\mu}+d+1, \widetilde{R})\right]_{m+d-k}\right)_{\alpha}^{N+1} \widetilde{t}_{d}^{\alpha} \frac{\partial \mathcal{F}^{o}}{\partial s_{k}}
\end{aligned}
$$




$$
\begin{aligned}
& +\sum_{k \geq 0} \sum_{1 \leq \alpha, \mu \leq N}(-1)^{k+1}\left(\left[P_{m}(\tilde{\mu}-k, \widetilde{R})\right]_{m-k}\right)_{\alpha}^{N+1} \eta^{\alpha \mu} \frac{\partial \mathcal{F}}{\partial t_{k}^{\mu}} \\
& +\sum_{d_{1}, d_{2} \geq 0} \sum_{1 \leq \alpha, \mu \leq N}(-1)^{d_{2}+1}\left(\left[P_{m}\left(\tilde{\mu}-d_{2}, \widetilde{R}\right)\right]_{m-1-d_{1}-d_{2}}\right)_{\alpha}^{N+1} \frac{\partial \mathcal{F}^{o}}{\partial s_{d_{1}}} \eta^{\alpha \mu} \frac{\partial \mathcal{F}}{\partial t_{d_{2}}^{\mu}} \\
& +\sum_{d_{1} \geq-1, d_{2} \geq 0}(-1)^{d_{2}+1}\left(\left[P_{m}\left(\tilde{\mu}-d_{2}, \widetilde{R}\right)\right]_{m-1-d_{1}-d_{2}}\right)_{N+1}^{N+1} \Phi_{N+1, d_{1}}^{\text {top }} \mathcal{F}^{N+1, d_{2}} \text {. }
\end{aligned}
$$

The first term here is equal to $\operatorname{Coef}_{\varepsilon^{-1}}\left(\frac{L_{m} e^{\varepsilon^{-2} \mathcal{F}+\varepsilon^{-1} \mathcal{F}^{o}}}{e^{\varepsilon^{-2} \mathcal{F}+\varepsilon^{-1} \mathcal{F}^{o}}}\right)$. The next four terms correspond to the four summations in the expression (3.5) for the operators $\mathcal{L}_{m}$. Since $\mu^{N+1}=\frac{1}{2}$, the last term is equal to zero. Thus,

$$
A_{m}^{N+1}=\operatorname{Coef}_{\varepsilon^{-1}}\left(\frac{\mathcal{L}_{m} e^{\varepsilon^{-2} \mathcal{F}+\varepsilon^{-1} \mathcal{F}^{o}}}{e^{\varepsilon^{-2} \mathcal{F}+\varepsilon^{-1} \mathcal{F} o}}\right)=0,
$$

that proves Theorem 3.4.

\section{Open Virasoro Constraints in All Genera}

There is a canonical construction that associates to a given semisimple conformal Frobenius manifold and its calibration a sequence of functions $\mathcal{F}_{0}\left(t_{*}^{*}\right)=$ $\mathcal{F}, \mathcal{F}_{1}\left(t_{*}^{*}\right), \mathcal{F}_{2}\left(t_{*}^{*}\right), \ldots$, such that for the differential operators $L_{m}$, given by (2.3), Eq. (1.5) hold (Givental 2001, 2004; Teleman 2012). If one considers the GromovWitten theory of a given target variety, then the functions $\mathcal{F}_{g}\left(t_{*}^{*}\right)$ are the generating series of intersection numbers on the moduli space of maps from a Riemann surface of genus $g$ to the target variety.

We conjecture that, under possibly some additional assumptions, there is a canonical way to associate to a solution $F^{o}$ of the WDVV equations, satisfying properties (1.8), (3.1), and its calibration a sequence of functions $\mathcal{F}_{0}^{o}\left(t_{*}^{\leq N}, s_{*}\right)=$ $\mathcal{F}^{o}, \mathcal{F}_{1}\left(t_{*}^{\leq N}, s_{*}\right), \mathcal{F}_{2}\left(t_{*}^{\leq N}, s_{*}\right), \ldots$, such that for the differential operators $\mathcal{L}_{m}$, given by (3.5), the equations

$$
\mathcal{L}_{m} e^{\sum_{g \geq 0} \varepsilon^{2 g-2} \mathcal{F}_{g}+\sum_{g \geq 0} \varepsilon^{g-1} \mathcal{F}_{g}^{o}}=0, \quad m \geq-1
$$

are satisfied. At the moment the conjecture is verified only in the case, corresponding to the intersection theory on the moduli space of Riemann surfaces with boundary (Pandharipande et al. 2014; Buryak and Tessler 2017).

As a step towards the proof of this conjecture, we verify the following commutation relations between the operators $\mathcal{L}_{m}$.

Proposition 5.1 We have $\left[\mathcal{L}_{m}, \mathcal{L}_{n}\right]=(m-n) \mathcal{L}_{m+n}, m, n \geq-1$. 
Proof Denote the parts of the expression for the operator $L_{m}$ from lines (2.3), (2.4) and (2.5) by $L_{m}^{1}, L_{m}^{2}$ and $L_{m}^{3}$, correspondingly. One can see that the operators $\mathcal{L}_{m}$ can be written in the following way:

$$
\begin{aligned}
\mathcal{L}_{m}= & L_{m}+\mathcal{L}_{m}^{1}+\mathcal{L}_{m}^{2}+\mathcal{L}_{m}^{3}+\mathcal{L}_{m}^{4}+\varepsilon^{-1} \delta_{m,-1} s+\delta_{m, 0} \frac{3}{4} \\
& +\sum_{d \geq 0} \frac{(d+m+1) !}{d !} s_{d} \frac{\partial}{\partial s_{d+m}}+\varepsilon \frac{3(m+1) !}{4} \frac{\partial}{\partial s_{m-1}}
\end{aligned}
$$

where

$$
\begin{aligned}
& \mathcal{L}_{m}^{1}=\varepsilon^{-1} \sum_{d \geq 0} \sum_{\substack{1 \leq \alpha \leq N \\
\mu^{\alpha}=-\frac{1}{2}-m-d}}\left(\prod_{i=0}^{m}(\widetilde{R}-i)\right)_{\alpha}^{N+1} \tilde{t}_{d}^{\alpha}, \\
& \mathcal{L}_{m}^{2}=\sum_{d, k \geq 0} \sum_{\substack{1 \leq \alpha \leq N \\
\mu^{\alpha}=\frac{1}{2}-m-d+k}}\left(\prod_{i=0}^{m}(\widetilde{R}+k+1-i)\right)_{\alpha}^{N+1} \tilde{t}_{d}^{\alpha} \frac{\partial}{\partial s_{k}}, \\
& \mathcal{L}_{m}^{3}=\varepsilon \sum_{k \geq 0} \sum_{\substack{1 \leq \alpha, \mu \leq N \\
\mu^{\alpha}=\frac{1}{2}-m+k}}(-1)^{k+1}\left(\prod_{i=0}^{m}(\widetilde{R}-i)\right)_{\alpha}^{N+1} \eta^{\alpha \mu} \frac{\partial}{\partial t_{k}^{\mu}}, \\
& \mathcal{L}_{m}^{4}=\varepsilon^{2} \sum_{d_{1}, d_{2} \geq 0} \sum_{\substack{1 \leq \alpha, \mu \leq N \\
\mu^{\alpha}=\frac{3}{2}-m+d_{1}+d_{2}}}(-1)^{d_{2}+1}\left(\prod_{\substack{1 \\
i=0}}^{m}\left(\widetilde{R}+d_{1}+1-i\right)\right)_{\alpha}^{N+1} \eta^{\alpha \mu} \frac{\partial^{2}}{\partial s_{d_{1}} \partial t_{d_{2}}^{\mu}} .
\end{aligned}
$$

Let us first prove that $\left[\mathcal{L}_{-1}, \mathcal{L}_{n}\right]=(-1-n) \mathcal{L}_{n-1}$, for $n \geq 0$. For this we compute

$$
\begin{aligned}
{\left[\mathcal{L}_{-1}, L_{n}\right]=} & (-1-n) L_{n-1}, \quad\left[\mathcal{L}_{-1}, \mathcal{L}_{n}^{1}\right]=\varepsilon^{-1} \sum_{d \geq 1} \sum_{\substack{1 \leq \alpha \leq N \\
\mu^{\alpha}=\frac{1}{2}-n-d}}\left(\prod_{i=0}^{n}(\widetilde{R}-i)\right)_{\alpha}^{N+1} \tilde{t}_{d}^{\alpha}, \\
{\left[\mathcal{L}_{-1}, \mathcal{L}_{n}^{2}\right]=} & \sum_{d \geq 1, k \geq 0} \sum_{\substack{1 \leq \alpha \leq N \\
\mu^{\alpha}=\frac{3}{2}-n-d+k}}\left(\prod_{i=0}^{n}(\widetilde{R}+k+1-i)\right)_{\alpha}^{N+1} \tilde{t}_{d}^{\alpha} \frac{\partial}{\partial s_{k}} \\
& -\varepsilon^{-1} \sum_{\substack{d \geq 0 \\
\mu^{\alpha}=\frac{1}{2}-n-d}} \sum_{\substack{1 \leq \alpha \leq N \\
i=0}}\left(\prod_{\alpha+1}^{n}(\widetilde{R}+1-i)\right)_{\alpha}^{N} \tilde{t}_{d}^{\alpha} \\
& -\sum_{\substack{1 \leq k \leq 0 \\
\mu^{\alpha}=\frac{3}{2}-n-d+k}}\left(\prod_{i=0}^{n}(\widetilde{R}+k+2-i)\right)_{\alpha}^{N+1} \tilde{t}_{d}^{\alpha} \frac{\partial}{\partial s_{k}}
\end{aligned}
$$




$$
\begin{aligned}
& {\left[\mathcal{L}_{-1}, \mathcal{L}_{n}^{3}\right]=\varepsilon^{-1} \sum_{\substack{1 \leq \alpha \leq N \\
\mu^{\alpha}=\frac{1}{2}-n}}\left(\prod_{i=0}^{n}(\widetilde{R}-i)\right)_{\alpha}^{N+1} \widetilde{t}_{0}^{\alpha}} \\
& +\varepsilon \sum_{k \geq 0} \sum_{\substack{1 \leq \alpha, \mu \leq N \\
\mu^{\alpha}=\frac{3}{2}-n+k}}(-1)^{k+1}\left(\prod_{i=0}^{n}(\widetilde{R}-i)\right)_{\alpha}^{N+1} \eta^{\alpha \mu} \frac{\partial}{\partial t_{k}^{\mu}} \\
& {\left[\mathcal{L}_{-1}, \mathcal{L}_{n}^{4}\right]=\varepsilon^{2} \sum_{d_{1}, d_{2} \geq 0} \sum_{\substack{1 \leq \alpha, \mu \leq N \\
\mu^{\alpha}=\frac{5}{2}-n+d_{1}+d_{2}}}(-1)^{d_{2}+1}\left(\prod_{i=0}^{n}\left(\widetilde{R}+d_{1}+1-i\right)\right)_{\alpha}^{N+1} \eta^{\alpha \mu} \frac{\partial^{2}}{\partial s_{d_{1}} \partial t_{d_{2}}^{\mu}}} \\
& +\sum_{d \geq 0} \sum_{\substack{1 \leq \alpha \leq N \\
\mu^{\alpha}=\frac{3}{2}-n+d}}\left(\prod_{i=0}^{n}(\widetilde{R}+d+1-i)\right)_{\alpha}^{N+1} \widetilde{t}_{0}^{\alpha} \frac{\partial}{\partial s_{d}} \\
& -\varepsilon \sum_{d \geq 0} \sum_{\substack{1 \leq \alpha, \mu \leq N \\
\mu^{\alpha}=\frac{3}{2}-n+d}}(-1)^{d+1}\left(\prod_{i=0}^{n}(\widetilde{R}+1-i)\right)_{\alpha}^{N+1} \eta^{\alpha \mu} \frac{\partial}{\partial t_{d}^{\mu}} \\
& -\varepsilon^{2} \sum_{d_{1}, d_{2} \geq 0} \sum_{\substack{1 \leq \alpha, \mu \leq N \\
\mu^{\alpha}=\frac{5}{2}-n+d_{1}+d_{2}}}(-1)^{d_{2}+1}\left(\prod_{i=0}^{n}\left(\widetilde{R}+d_{1}+2-i\right)\right)_{\alpha}^{N+1} \eta^{\alpha \mu} \frac{\partial^{2}}{\partial s_{d_{1}} \partial t_{d_{2}}^{\mu}},
\end{aligned}
$$

and

$$
\begin{aligned}
{\left[\mathcal{L}_{-1}, \sum_{d \geq 0} \frac{(d+n+1) !}{d !} s_{d} \frac{\partial}{\partial s_{d+n}}\right] } & =-\delta_{n, 0} \varepsilon^{-1} s+(-1-n) \sum_{d \geq 0} \frac{(d+n) !}{d !} s_{d} \frac{\partial}{\partial s_{d+n-1}}, \\
{\left[\mathcal{L}_{-1}, \varepsilon \frac{3(n+1) !}{4} \frac{\partial}{\partial s_{n-1}}\right] } & =-\delta_{n, 1} \frac{3}{2}-\varepsilon \frac{3(n+1) !}{4} \frac{\partial}{\partial s_{n-2}} .
\end{aligned}
$$

It remains to note that the sum of the expressions in lines $(5.1),(5.3),(5.5)$ is equal to $(-1-n) \mathcal{L}_{n-1}^{1}$, the sum of the expressions in lines $(5.2),(5.4),(5.8)$ is equal to $(-1-n) \mathcal{L}_{n-1}^{2}$, the sum of the expressions in lines (5.6), (5.9) is equal to $(-1-n) \mathcal{L}_{n-1}^{3}$ and the sum of the expressions in lines $(5.7),(5.10)$ is equal to $(-1-n) \mathcal{L}_{n-1}^{4}$.

Let us now prove the proposition for $m, n \geq 0$. The commutator $\left[\mathcal{L}_{m}, \mathcal{L}_{n}\right]$ has the form

$$
\begin{aligned}
{\left[\mathcal{L}_{m}, \mathcal{L}_{n}\right]=} & (m-n) L_{m+n}+\varepsilon^{-1} \sum_{d \geq 0} \sum_{1 \leq \alpha \leq N} A_{\alpha, d} \widetilde{t}_{d}^{\alpha} \\
& +\left(\sum_{p, q \geq 0} \sum_{1 \leq \alpha \leq N} B_{\alpha, p}^{q} \widetilde{t}_{p}^{\alpha} \frac{\partial}{\partial s_{q}}+\sum_{p, q \geq 0} C_{p}^{q} s_{p} \frac{\partial}{\partial s_{q}}+D\right)
\end{aligned}
$$




$$
\begin{aligned}
& +\varepsilon\left(\sum_{d \geq 0} \sum_{1 \leq \alpha \leq N} E^{\alpha, d} \frac{\partial}{\partial t_{d}^{\alpha}}+\sum_{d \geq 0} G^{d} \frac{\partial}{\partial s_{d}}\right) \\
& +\varepsilon^{2}\left(\sum_{p, q \geq 0} \sum_{1 \leq \alpha \leq N} H^{\alpha, p ; q} \frac{\partial}{\partial t_{p}^{\alpha} \partial s_{q}}+\sum_{p, q \geq 0} I^{p ; q} \frac{\partial}{\partial s_{p} \partial s_{q}}\right) .
\end{aligned}
$$

Let us consider separately the terms on the right-hand side of this expression.

1. Term $\varepsilon^{-1} \sum_{d \geq 0} \sum_{1 \leq \alpha \leq N} A_{\alpha, d} \widetilde{t}_{d}^{\alpha}$ :

$$
\varepsilon^{-1} \sum_{d \geq 0} \sum_{1 \leq \alpha \leq N} A_{\alpha, d} \widetilde{t}_{d}^{\alpha}=\left[L_{m}^{2}, \mathcal{L}_{n}^{1}\right]+\left[L_{m}^{1}, \mathcal{L}_{n}^{3}\right]-\left[L_{n}^{2}, \mathcal{L}_{m}^{1}\right]-\left[L_{n}^{1}, \mathcal{L}_{m}^{3}\right]
$$

We compute

$$
\left[L_{m}^{2}, \mathcal{L}_{n}^{1}\right]=\varepsilon^{-1} \sum_{d, k \geq 0} \sum_{\substack{1 \leq \alpha \leq N \\ \mu^{\alpha}=-\frac{1}{2}-m-n-d}} \sum_{\substack{1 \leq \beta \leq N \\ \mu^{\beta}=-\frac{1}{2}-n-k}}\left(\prod_{i=0}^{m}(R-n-i)\right)_{\alpha}^{\beta}\left(\prod_{i=0}^{n}(\widetilde{R}-i)\right)_{\beta}^{N+1} \tilde{t}_{d}^{\alpha} .
$$

Similarly, we get

$$
\left[L_{m}^{1}, \mathcal{L}_{n}^{3}\right]=\varepsilon^{-1} \sum_{d \geq 0, k<0} \sum_{\substack{1 \leq \alpha \leq N \\ \mu^{\alpha}=-\frac{1}{2}-m-n-d}} \sum_{\substack{1 \leq \beta \leq N \\ 1 \\ 1 \\=-\frac{1}{2}-n-k}}\left(\prod_{i=0}^{m}(R-n-i)\right)_{\alpha}^{\beta}\left(\prod_{i=0}^{n}(\widetilde{R}-i)\right)_{\beta}^{N+1} \tilde{t}_{d}^{\alpha} .
$$

As a result,

$$
\left[L_{m}^{2}, \mathcal{L}_{n}^{1}\right]+\left[L_{m}^{1}, \mathcal{L}_{n}^{3}\right]=\varepsilon^{-1} \sum_{d \geq 0} \sum_{\substack{1 \leq \alpha \leq N \\ \mu^{\alpha}=-\frac{1}{2}-m-n-d}}\left((\widetilde{R}-n) \prod_{i=0}^{m+n}(\widetilde{R}-i)\right)_{\alpha}^{N+1} \widetilde{t}_{d}^{\alpha},
$$

which finally gives

$$
\begin{aligned}
& {\left[L_{m}^{2}, \mathcal{L}_{n}^{1}\right]+\left[L_{m}^{1}, \mathcal{L}_{n}^{3}\right]-\left[L_{n}^{2}, \mathcal{L}_{m}^{1}\right]-\left[L_{n}^{1}, \mathcal{L}_{m}^{3}\right]} \\
& =(m-n) \varepsilon^{-1} \sum_{d \geq 0} \sum_{\substack{1 \leq \alpha \leq N \\
\mu^{\alpha}=-\frac{1}{2}-m-n-d}}\left(\prod_{i=0}^{m+n}(\widetilde{R}-i)\right)_{\alpha}^{N+1} \widetilde{t}_{d}^{\alpha}=(m-n) \mathcal{L}_{m+n}^{1},
\end{aligned}
$$

as required. 
2. Term $\sum_{p, q \geq 0} \sum_{1 \leq \alpha \leq N} B_{\alpha, p}^{q} \widetilde{t}_{p}^{\alpha} \frac{\partial}{\partial s_{q}}$ :

$$
\begin{aligned}
\sum_{p, q \geq 0} \sum_{1 \leq \alpha \leq N} B_{\alpha, p}^{q} \tilde{t}_{p}^{\alpha} \frac{\partial}{\partial s_{q}}= & {\left[L_{m}^{2}, \mathcal{L}_{n}^{2}\right]+\left[\mathcal{L}_{m}^{2}, \sum_{d \geq 0} \frac{(d+n+1) !}{d !} s_{d} \frac{\partial}{\partial s_{d+n}}\right] } \\
& +\left[L_{m}^{1}, \mathcal{L}_{n}^{4}\right]- \\
& -\left[L_{n}^{2}, \mathcal{L}_{m}^{2}\right]-\left[\mathcal{L}_{n}^{2}, \sum_{d \geq 0} \frac{(d+m+1) !}{d !} s_{d} \frac{\partial}{\partial s_{d+m}}\right] \\
& -\left[L_{n}^{1}, \mathcal{L}_{m}^{4}\right] .
\end{aligned}
$$

We compute

$$
\begin{aligned}
& {\left[L_{m}^{2}, \mathcal{L}_{n}^{2}\right]+\left[\mathcal{L}_{m}^{2}, \sum_{d \geq 0} \frac{(d+n+1) !}{d !} s_{d} \frac{\partial}{\partial s_{d+n}}\right]} \\
& =\sum_{d, k, l \geq 0} \sum_{\substack{1 \leq \alpha \leq N \\
\mu^{\alpha}=\frac{1}{2}-m-n-d+k}} \sum_{\substack{1 \leq \beta \leq N+1 \\
\mu^{\beta}=\frac{1}{2}-n+k-l}} \\
& \times\left(\prod_{i=0}^{m}(\widetilde{R}+k+1-n-i)\right)_{\alpha}^{\beta}\left(\prod_{i=0}^{n}(\widetilde{R}+k+1-i)\right)_{\beta}^{N+1} \widetilde{t}_{d}^{\alpha} \frac{\partial}{\partial s_{k}},
\end{aligned}
$$

and then check that this sum, after replacing the summation $\sum_{l \geq 0}$ by the summation $\sum_{l<0}$, is equal to the commutator $\left[L_{m}^{1}, \mathcal{L}_{n}^{4}\right]$. This gives

$$
\begin{aligned}
& {\left[L_{m}^{2}, \mathcal{L}_{n}^{2}\right]+\left[\mathcal{L}_{m}^{2}, \sum_{d \geq 0} \frac{(d+n+1) !}{d !} s_{d} \frac{\partial}{\partial s_{d+n}}\right]+\left[L_{m}^{1}, \mathcal{L}_{n}^{4}\right]} \\
& =\sum_{d, k \geq 0} \sum_{\substack{1 \leq \alpha \leq N \\
\mu^{\alpha}=\frac{1}{2}-m-n-d+k}}\left((\widetilde{R}+k+1-n) \prod_{i=0}^{m+n}(\widetilde{R}+k+1-i)\right)_{\alpha}^{N+1} \widetilde{t}_{d}^{\alpha} \frac{\partial}{\partial s_{k}} .
\end{aligned}
$$

As a result, $\sum_{p, q \geq 0} \sum_{1 \leq \alpha \leq N} B_{\alpha, p}^{q} \widetilde{t}_{p}^{\alpha} \frac{\partial}{\partial s_{q}}=(m-n) \mathcal{L}_{m+n}^{2}$, as required.

3. Term $\sum_{p, q \geq 0} C_{p}^{q} s_{p} \frac{\partial}{\partial s_{q}}$ :

$$
\begin{aligned}
\sum_{p, q \geq 0} C_{p}^{q} s_{p} \frac{\partial}{\partial s_{q}} & =\left[\sum_{p \geq 0} \frac{(p+m+1) !}{p !} s_{p} \frac{\partial}{\partial s_{p+m}}, \sum_{q \geq 0} \frac{(q+n+1) !}{q !} s_{q} \frac{\partial}{\partial s_{q+n}}\right] \\
& =(m-n) \sum_{d \geq 0} \frac{(d+m+n+1) !}{d !} s_{d} \frac{\partial}{\partial s_{d+m+n}},
\end{aligned}
$$


as required.

4. Constant D:

$$
\begin{aligned}
D & =\left[\mathcal{L}_{m}^{1}, \mathcal{L}_{n}^{3}\right]-\left[\mathcal{L}_{n}^{1}, \mathcal{L}_{m}^{3}\right], \quad \text { where } \\
{\left[\mathcal{L}_{m}^{1}, \mathcal{L}_{n}^{3}\right] } & =\sum_{\substack { d \geq 0 \\
\begin{subarray}{c}{1 \leq \alpha, \beta \leq N \\
\mu^{\alpha}=-\frac{1}{2}-m-d \\
\mu^{\beta}=\frac{1}{2}-n+d{ d \geq 0 \\
\begin{subarray} { c } { 1 \leq \alpha , \beta \leq N \\
\mu ^ { \alpha } = - \frac { 1 } { 2 } - m - d \\
\mu ^ { \beta } = \frac { 1 } { 2 } - n + d } }\end{subarray}}\left(\prod_{i=0}^{m}(\widetilde{R}-i)\right)_{\alpha}^{N+1} \eta^{\alpha \beta}\left(\prod_{i=0}^{n}(\widetilde{R}-i)\right)_{\beta}^{N+1}
\end{aligned}
$$

Because of the property $\mu \eta+\eta \mu=0$, the last expression is equal to zero unless $m+n=0$, which implies that $m=n=0$. Thus, $D=0$, as required.

5. Term $\varepsilon \sum_{d \geq 0} \sum_{1 \leq \alpha \leq N} E^{\alpha, d} \frac{\partial}{\partial t_{d}^{\alpha}}$ :

$$
\varepsilon \sum_{d \geq 0} \sum_{1 \leq \alpha \leq N} E^{\alpha, d} \frac{\partial}{\partial t_{d}^{\alpha}}=\left[L_{m}^{2}, \mathcal{L}_{n}^{3}\right]+\left[L_{m}^{3}, \mathcal{L}_{n}^{1}\right]-\left[L_{n}^{2}, \mathcal{L}_{m}^{3}\right]-\left[L_{n}^{3}, \mathcal{L}_{m}^{1}\right]
$$

We first compute

$$
\begin{aligned}
{\left[L_{m}^{2}, \mathcal{L}_{n}^{3}\right]=\varepsilon \sum_{d, k \geq 0} \sum_{\begin{array}{c}
1 \leq \alpha, \beta, \gamma \leq N \\
\mu^{\beta}=m+n-k-\frac{1}{2} \\
\mu^{\alpha}=-\frac{1}{2}+n-d
\end{array}}(-1)^{d}\left(\prod_{i=0}^{n}(\widetilde{R}-i)\right)_{\gamma}^{N+1} \eta^{\gamma \alpha}\left(\prod_{i=0}^{m}(R+n+i)\right)_{\alpha}^{\beta} \frac{\partial}{\partial t_{k}^{\beta}} } \\
=\varepsilon \sum_{d, k \geq 0} \sum_{\substack{1 \leq \alpha, \beta, \gamma \leq N \\
\mu^{\beta}=m+n-k-\frac{1}{2} \\
\mu^{\gamma}=\frac{1}{2}-n+d}}(-1)^{k+1}\left(\prod_{i=0}^{n}(\widetilde{R}-i)\right)_{\gamma}^{N+1}\left(\prod_{i=0}^{m}(R-n-i)\right)_{\alpha}^{\gamma} \eta^{\alpha \beta} \frac{\partial}{\partial t_{k}^{\beta}},
\end{aligned}
$$

where the second equality is obtained using the property $\eta R_{i} \eta^{-1}=(-1)^{i-1} R_{i}^{T}$. Then one can compute that the commutator $\left[L_{m}^{3}, \mathcal{L}_{n}^{1}\right]$ is equal to the expression in line (5.11) with the summation $\sum_{d \geq 0}$ replaced by $\sum_{d<0}$. This implies that

$$
\begin{gathered}
{\left[L_{m}^{2}, \mathcal{L}_{n}^{3}\right]+\left[L_{m}^{3}, \mathcal{L}_{n}^{1}\right]=\varepsilon \sum_{k \geq 0} \sum_{\substack{1 \leq \alpha, \beta \leq N \\
\mu^{\beta}=m+n-k-\frac{1}{2}}}(-1)^{k+1}} \\
\quad\left(\prod_{i=0}^{n}(\widetilde{R}-i) \prod_{i=0}^{m}(\widetilde{R}-n-i)\right)_{\alpha}^{N+1} \eta^{\alpha \beta} \frac{\partial}{\partial t_{k}^{\beta}},
\end{gathered}
$$


and, as a result,

$$
\begin{aligned}
& {\left[L_{m}^{2}, \mathcal{L}_{n}^{3}\right]+\left[L_{m}^{3}, \mathcal{L}_{n}^{1}\right]-\left[L_{n}^{2}, \mathcal{L}_{m}^{3}\right]-\left[L_{n}^{3}, \mathcal{L}_{m}^{1}\right]} \\
& =(m-n) \varepsilon \sum_{k \geq 0} \sum_{\substack{1 \leq \alpha, \beta \leq N \\
\mu^{\beta}=m+n-k-\frac{1}{2}}}(-1)^{k+1}\left(\prod_{i=0}^{m+n}(\widetilde{R}-i)\right)_{\alpha}^{N+1} \eta^{\alpha \beta} \frac{\partial}{\partial t_{k}^{\beta}}=(m-n) \mathcal{L}_{m+n}^{3},
\end{aligned}
$$

as required.

6. Term $\varepsilon \sum_{d \geq 0} G^{d} \frac{\partial}{\partial s_{d}}$ :

$$
\begin{aligned}
\varepsilon \sum_{d \geq 0} G^{d} \frac{\partial}{\partial s_{d}}= & (m-n) \varepsilon \frac{3(m+n+1) !}{4} \frac{\partial}{\partial s_{m+n-1}} \\
& +\left[\mathcal{L}_{m}^{2}, \mathcal{L}_{n}^{3}\right]+\left[\mathcal{L}_{m}^{1}, \mathcal{L}_{n}^{4}\right]-\left[\mathcal{L}_{n}^{2}, \mathcal{L}_{m}^{3}\right]-\left[\mathcal{L}_{n}^{1}, \mathcal{L}_{m}^{4}\right] .
\end{aligned}
$$

We compute

$$
\begin{aligned}
{\left[\mathcal{L}_{m}^{2}, \mathcal{L}_{n}^{3}\right]=} & \varepsilon \sum_{d \geq 0}(-1)^{d} \sum_{\substack{1 \leq \alpha, \beta \leq N \\
\mu^{\alpha}=-\frac{1}{2}+n-d \\
\mu^{\beta}=\frac{1}{2}-n+d}} \\
& \times\left(\prod_{i=0}^{m}(\widetilde{R}+m+n-i)\right)_{\alpha}^{N+1} \eta^{\alpha \beta}\left(\prod_{i=0}^{n}(\widetilde{R}-i)\right)_{\beta}^{N+1} \frac{\partial}{\partial s_{m+n-1}}
\end{aligned}
$$

A term in this sum is equal to zero unless $\mu^{\alpha}<\frac{1}{2} \Leftrightarrow d>n-1$ and $\mu^{\beta}<$ $\frac{1}{2} \Leftrightarrow d<n$, that never happens. Therefore, $\left[\mathcal{L}_{m}^{2}, \mathcal{L}_{n}^{3}\right]=0$, and, similarly, one can check that $\left[\mathcal{L}_{m}^{1}, \mathcal{L}_{n}^{4}\right]=\left[\mathcal{L}_{n}^{2}, \mathcal{L}_{m}^{3}\right]=\left[\mathcal{L}_{n}^{1}, \mathcal{L}_{m}^{4}\right]=0$. Hence, $\varepsilon \sum_{d \geq 0} G^{d} \frac{\partial}{\partial s_{d}}=$ $(m-n) \varepsilon \frac{3(m+n+1) !}{4} \frac{\partial}{\partial s_{m+n-1}}$, as required.

7. Term $\varepsilon^{2} \sum_{p, q \geq 0} \sum_{1 \leq \alpha \leq N} H^{\alpha, p ; q} \frac{\partial}{\partial t_{p}^{\alpha} \partial s_{q}}$ :

$$
\begin{aligned}
& \varepsilon^{2} \sum_{p, q \geq 0} \sum_{1 \leq \alpha \leq N} H^{\alpha, p ; q} \frac{\partial}{\partial t_{p}^{\alpha} \partial s_{q}} \\
& =\left[L_{m}^{2}, \mathcal{L}_{n}^{4}\right]+\left[L_{m}^{3}, \mathcal{L}_{n}^{2}\right]+\left[\mathcal{L}_{m}^{4}, \sum_{d \geq 0} \frac{(d+n+1) !}{d !} s_{d} \frac{\partial}{\partial s_{d+n}}\right] \\
& -\left[L_{n}^{2}, \mathcal{L}_{m}^{4}\right]-\left[L_{n}^{3}, \mathcal{L}_{m}^{2}\right]-\left[\mathcal{L}_{n}^{4}, \sum_{d \geq 0} \frac{(d+m+1) !}{d !} s_{d} \frac{\partial}{\partial s_{d+m}}\right] .
\end{aligned}
$$


We proceed with the computation

$$
\begin{aligned}
{\left[L_{m}^{2}, \mathcal{L}_{n}^{4}\right]=} & \varepsilon^{2} \sum_{d_{1}, d_{2}, d \geq 0}(-1)^{d} \sum_{\begin{array}{c}
1 \leq \alpha, \beta, \mu \leq N \\
\mu^{\alpha}=\frac{3}{2}-n+d_{1}+d \\
\mu^{\beta}=-\frac{3}{2}+m+n-d_{1}-d_{2}
\end{array}}\left(\prod_{i=0}^{n}\left(\widetilde{R}+d_{1}+1-i\right)\right)_{\alpha}^{N+1} \\
& \times \eta^{\alpha \mu}\left(\prod_{i=0}^{m}\left(R-1-d_{1}+n+i\right)\right)_{\mu}^{\beta} \frac{\partial^{2}}{\partial s_{d_{1}} \partial t_{d_{2}}^{\beta}}
\end{aligned}
$$

Using the relation $\eta R_{i} \eta^{-1}=(-1)^{i-1} R_{i}^{T}$, we convert this sum to

$$
\begin{aligned}
& \varepsilon^{2} \sum_{d_{1}, d_{2}, d \geq 0}(-1)^{d_{2}+1} \sum_{\substack{1 \leq \alpha \leq N+1 \\
\mu^{\alpha}=\frac{3}{2}-n+d_{1}+d \\
\mu^{\beta}=-\frac{3}{2}+m+n-d_{1}-d_{2}}}^{N+1}\left(\prod_{\alpha}^{m}\left(\widetilde{R}+1+d_{1}-n-i\right)\right)_{\mu}^{\alpha} \eta^{\mu \beta} \frac{\partial^{2}}{\partial s_{d_{1}} \partial t_{d_{2}}^{\beta}} .
\end{aligned}
$$

Then one can check that the expression $\left[L_{m}^{3}, \mathcal{L}_{n}^{2}\right]+\left[\mathcal{L}_{m}^{4}, \sum_{d \geq 0} \frac{(d+n+1) !}{d !} s_{d} \frac{\partial}{\partial s_{d+n}}\right]$ is equal to the expression (5.12), with the summation $\sum_{d \geq 0}$ replaced by $\sum_{d<0}$. As a result,

$$
\begin{aligned}
& {\left[L_{m}^{2}, \mathcal{L}_{n}^{4}\right]+\left[L_{m}^{3}, \mathcal{L}_{n}^{2}\right]+\left[\mathcal{L}_{m}^{4}, \sum_{d \geq 0} \frac{(d+n+1) !}{d !} s_{d} \frac{\partial}{\partial s_{d+n}}\right]} \\
& =\varepsilon^{2} \sum_{d_{1}, d_{2} \geq 0}(-1)^{d_{2}+1} \sum_{\mu^{\beta}=-\frac{3}{2}+m+n-d_{1}-d_{2}}\left(\left(\widetilde{R}+d_{1}+1-n\right) \prod_{i=0}^{m+n}\left(\widetilde{R}+d_{1}+1-i\right)\right)_{\mu}^{N+1} \\
& \quad \times \eta^{\mu \beta} \frac{\partial^{2}}{\partial s_{d_{1}} \partial t_{d_{2}}^{\beta}},
\end{aligned}
$$

which gives $\varepsilon^{2} \sum_{p, q \geq 0} \sum_{1 \leq \alpha \leq N} H^{\alpha, p ; q} \frac{\partial}{\partial t_{p}^{\alpha} \partial s_{q}}=(m-n) \mathcal{L}_{m+n}^{4}$, as required.

8. Term $\varepsilon^{2} \sum_{p, q \geq 0} I^{p ; q} \frac{\partial^{2}}{\partial s_{p} \partial s_{q}}$ :

$$
\varepsilon^{2} \sum_{p, q \geq 0} I^{p ; q} \frac{\partial^{2}}{\partial s_{p} \partial s_{q}}=\left[\mathcal{L}_{m}^{4}, \mathcal{L}_{n}^{2}\right]-\left[\mathcal{L}_{n}^{4}, \mathcal{L}_{m}^{2}\right]
$$


where

$$
\begin{aligned}
{\left[\mathcal{L}_{m}^{4}, \mathcal{L}_{n}^{2}\right]=} & \varepsilon^{2} \sum_{p, q, d \geq 0} \sum_{\substack{1 \leq \alpha, \beta \leq N \\
\mu^{\alpha}=\frac{3}{2}-m+p+d \\
\mu^{\beta}=\frac{1}{2}-n+q-d}}(-1)^{d+1}\left(\prod_{i=0}^{m}(\widetilde{R}+p+1-i)\right)_{\alpha}^{N+1} \\
& \times \eta^{\alpha \beta}\left(\prod_{i=0}^{n}(\widetilde{R}+q+1-i)\right)_{\beta}^{N+1} \frac{\partial^{2}}{\partial s_{p} \partial s_{q}} .
\end{aligned}
$$

A term in this sum is equal to zero unless $\mu^{\alpha}<\frac{1}{2} \Leftrightarrow p+d \leq m-2, \mu^{\beta}<$ $\frac{1}{2} \Leftrightarrow q-d \leq n-1$ and $\mu^{\alpha}+\mu^{\beta}=0 \Leftrightarrow p+q=m+n-2$. The first two condition give $p+q \leq m+n-3$, that contradicts the third condition. Thus, $\varepsilon^{2} \sum_{p, q \geq 0} I{ }^{p ; q} \frac{\partial^{2}}{\partial s_{p} \partial s_{q}}=0$, as required. This completes the proof of the proposition.

\section{Examples of Solutions of the Open WDVV Equations}

In this section we present several examples of solutions of the open WDVV equations, satisfying conditions (1.8) and (3.1) and, thus, Theorem 3.4 can be applied to them.

\subsection{Extended $r$-Spin Theory}

Let us fix an integer $r \geq 2$. There is a conformal Frobenius manifold that controls the integrals of the so-called Witten class over the moduli space of stable curves of genus 0 with an $r$-spin structure. This Frobenius manifold has dimension $r-1$ and is described by a potential $F^{r \text {-spin }}\left(t^{1}, \ldots, t^{r-1}\right)$ with the metric $\eta$, given by $\eta_{\alpha \beta}=\delta_{\alpha+\beta, r}$, and the Euler vector field

$$
E=\sum_{\alpha=1}^{r-1} \frac{r+1-\alpha}{r} t^{\alpha} \frac{\partial}{\partial t^{\alpha}}, \quad \sum_{\alpha=1}^{r-1} E^{\alpha} \frac{\partial F^{r} \text {-spin }}{\partial t^{\alpha}}=\frac{2 r+2}{r} F^{r} \text {-spin. }
$$

The conformal dimension is $\delta=\frac{r-2}{r}$. The potential $F^{r \text {-spin }}$ is a polynomial in the variables $t^{1}, \ldots, t^{r-1}$. For more details, we refer a reader, for example, to Pandharipande et al. (2019), Buryak et al. (2019).

The generating series of the descendent integrals with Witten's class over the moduli space of curves of genus 0 with an $r$-spin structure gives the descendent potential $\mathcal{F}^{r \text {-spin }}\left(t_{*}^{*}\right)$, corresponding to our Frobenius potential $F^{r \text {-spin }}$. This descendent potential corresponds to a calibration with all the matrices $R_{i}$ being zero. Thus, the Virasoro operators $L_{m}$ are given by 


$$
\begin{aligned}
L_{m}= & \sum_{\substack{1 \leq \alpha \leq r-1 \\
a \geq 0}} \frac{(\alpha+a r)^{\overline{m+1}}}{r^{m+1}}\left(t_{a}^{\alpha}-\delta^{\alpha, 1} \delta_{a, 1}\right) \frac{\partial}{\partial t_{a+m}^{\alpha}}+\frac{\varepsilon^{2}}{2} \sum_{\substack{\alpha+\beta=r \\
a+b=m-1}} \frac{\alpha^{\overline{a+1}} \beta^{\overline{b+1}}}{r^{m+1}} \frac{\partial^{2}}{\partial t_{a}^{\alpha} \partial t_{b}^{\beta}} \\
& +\delta_{m,-1} \frac{1}{2 \varepsilon^{2}} \sum_{\alpha+\beta=r} t_{0}^{\alpha} t_{0}^{\beta}+\delta_{m, 0} \frac{r^{2}-1}{24 r},
\end{aligned}
$$

where we use the notation

$$
x^{\bar{n}}:= \begin{cases}\prod_{i=1}^{n}(x+i-1), & \text { if } n \geq 1 \\ 1, & \text { if } n=0\end{cases}
$$

for a complex number $x$ and an integer $n \geq 0$.

In Jarvis et al. (2001) the authors considered a generalization of the $r$-spin theory, that we call the extended $r$-spin theory, and in Buryak et al. (2019) the authors noticed (see Remark 1.1) that such a generalization produces a solution $F^{\mathrm{ext}}\left(t^{1}, \ldots, t^{r}\right)$ of the open WDVV equations, satisfying condition (1.8) and the homogeneity condition

$$
\sum_{\alpha=1}^{r-1} E^{\alpha} \frac{\partial F^{\mathrm{ext}}}{\partial t^{\alpha}}+\frac{1-\delta}{2} t^{r} \frac{\partial F^{\mathrm{ext}}}{\partial t^{r}}=\frac{3-\delta}{2} F^{\mathrm{ext}}
$$

Recall that we identify $t^{r}=s$. Note that the function $F^{\text {ext }}$ also controls the open $r$ spin theory, constructed in [BCT18]. The generating series $\mathcal{F}^{\text {ext }}\left(t_{*}^{*}\right)$ of the descendent integrals in the extended $r$-spin theory is the open descendent potential, corresponding to the function $F^{\text {ext }}$ and a calibration with all the matrices $\widetilde{R}_{i}$ being zero. Thus, the associated open Virasoro operators $\mathcal{L}_{m}$ are

$\mathcal{L}_{m}=L_{m}+\sum_{d \geq 0} \frac{(d+m+1) !}{d !} t_{d}^{r} \frac{\partial}{\partial t_{d+m}^{r}}+\varepsilon \frac{3(m+1) !}{4} \frac{\partial}{\partial t_{m-1}^{r}}+\delta_{m,-1} \varepsilon^{-1} t^{r}+\delta_{m, 0} \frac{3}{4}$

\subsection{Solutions Given by the Canonical Coordinates}

Consider a conformal Frobenius manifold given by a potential $F=F\left(t^{1}, \ldots, t^{N}\right)$, a metric $\eta$ and an Euler vector field $E$. Suppose that the Frobenius manifold is semisimple and let $u^{1}, \ldots, u^{N}$ be the canonical coordinates. It is well-known that in the canonical coordinates the Euler vector field $E$ looks as

$$
E=\sum_{i=1}^{N}\left(u^{i}+a^{i}\right) \frac{\partial}{\partial u^{i}}
$$

for some constants $a^{i} \in \mathbb{C}$. 
Proposition 6.1 For any $1 \leq k \leq N$ the function $F^{o}=u^{k} \cdot s$ satisfies the open WDVV equations together with condition (1.8) and the homogeneity condition

$$
\sum_{\alpha=1}^{N} E^{\alpha} \frac{\partial F^{o}}{\partial t^{\alpha}}+\frac{1-\delta}{2} s \frac{\partial F^{o}}{\partial s}=\frac{3-\delta}{2} F^{o}+a^{k} s
$$

Proof Recall that the canonical coordinates satisfy the equations

$$
c_{\alpha \beta}^{v} \frac{\partial u^{k}}{\partial t^{\nu}}=\frac{\partial u^{k}}{\partial t^{\alpha}} \frac{\partial u^{k}}{\partial t^{\beta}}, \quad 1 \leq \alpha, \beta \leq N,
$$

which immediately imply Eq. (1.7). Equation (1.6) for the function $F^{o}=u \cdot s$ are equivalent to

$$
c_{\alpha \beta}^{\nu} \frac{\partial^{2} u^{k}}{\partial t^{\nu} \partial t^{\gamma}}+\frac{\partial^{2} u^{k}}{\partial t^{\alpha} \partial t^{\beta}} \frac{\partial u^{k}}{\partial t^{\gamma}}=c_{\gamma \beta}^{\nu} \frac{\partial^{2} u^{k}}{\partial t^{\nu} \partial t^{\alpha}}+\frac{\partial^{2} u^{k}}{\partial t^{\gamma} \partial t^{\beta}} \frac{\partial u^{k}}{\partial t^{\alpha}} .
$$

Differentiating Eq. (6.2) with respect to $t^{\gamma}$, we get

$$
\frac{\partial c_{\alpha \beta}^{\nu}}{\partial t^{\gamma}} \frac{\partial u^{k}}{\partial t^{\nu}}=\frac{\partial^{2} u^{k}}{\partial t^{\gamma} \partial t^{\alpha}} \frac{\partial u^{k}}{\partial t^{\beta}}+\frac{\partial u^{k}}{\partial t^{\alpha}} \frac{\partial^{2} u^{k}}{\partial t^{\gamma} \partial t^{\beta}}-c_{\alpha \beta}^{\nu} \frac{\partial^{2} u^{k}}{\partial t^{\nu} \partial t^{\gamma}} .
$$

Combining this equation with the similar equation for $\frac{\partial c_{\gamma \beta}^{v}}{\partial t^{\alpha}} \frac{\partial u^{k}}{\partial t^{\nu}}$ and noting that $\frac{\partial c_{\gamma \beta}^{v}}{\partial t^{\alpha}}=\frac{\partial c_{\alpha \beta}^{\nu}}{\partial t^{\gamma}}$, we get Eq. (6.3).

Property (1.8) follows from the fact that $\frac{\partial}{\partial t^{1}}=\sum_{i=1}^{n} \frac{\partial}{\partial u^{i}}$. The homogeneity property (6.1) is obvious.

\subsection{Open Gromov-Witten Theory of $\mathbb{P}^{1}$}

Consider the 2-dimensional Frobenius manifold given by the Gromov-Witten theory of $\mathbb{P}^{1}$ :

$$
F\left(t_{1}, t_{2}\right)=\frac{1}{2} t_{1}^{2} t_{2}+e^{t_{2}}
$$

The Euler vector field is $E=t_{1} \frac{\partial}{\partial t_{1}}+2 \frac{\partial}{\partial t_{2}}$ and $\delta=1$. Let us find all solutions $F^{o}\left(t_{1}, t_{2}, s\right)$ of the open WDVV Eqs. (1.6), (1.7), satisfying condition (1.8) and the homogeneity condition

$$
t_{1} \frac{\partial F^{o}}{\partial t_{1}}+2 \frac{\partial F^{o}}{\partial t_{2}}=F^{o}+D_{1} t_{1}+D_{2} t_{2}+\widetilde{D} s+E .
$$

We consider such solutions up to adding a constant and linear terms in the variables $t_{1}, t_{2}$ and $s$. Then we can assume that $D_{1}=0$. The general form of such a function $F^{o}$ 
is $F^{o}=t_{1} s+e^{\frac{t_{2}}{2}} \phi\left(t_{2}, s\right)$, for some function $\phi\left(t_{2}, s\right)$, satisfying $2 e^{\frac{t_{2}}{2}} \frac{\partial \phi}{\partial t_{2}}=D_{2} t_{2}+$ $\widetilde{D} s+E$. Making the transformation $\phi \mapsto \phi-\left(D_{2}\left(t_{2}+2\right)+\widetilde{D} s+E\right) e^{-\frac{t_{2}}{2}}$, we come to a function $F^{o}$ of the form

$$
F^{o}=t_{1} s+e^{\frac{t_{2}}{2}} \phi(s)
$$

where the function $\phi$ depends only on $s$. Such a function $F^{o}$ satisfies the homogeneity property

$$
t_{1} \frac{\partial F^{o}}{\partial t_{1}}+2 \frac{\partial F^{o}}{\partial t_{2}}=F^{o}
$$

and condition (1.8).

The system of open WDVV Eqs. (1.6), (1.7) for a function $F^{o}$ of the form (6.4) is equivalent to the equation $\left(\phi^{\prime}\right)^{2}-\phi \phi^{\prime \prime}=4$ and, solving this ordinary differential equation, we get the following two-parameter family of solutions:

$$
F_{\alpha, \beta}^{o}=t_{1} s \pm 2 \alpha^{-1} e^{\frac{t_{2}}{2}} \sinh (\alpha(s+\beta)), \quad \alpha, \beta \in \mathbb{C}
$$

For $\alpha=0$ we get the functions

$$
F_{0, \beta}^{o}=\left.\left(t_{1} s \pm 2 \alpha^{-1} e^{\frac{t_{2}}{2}} \sinh (\alpha(s+\beta))\right)\right|_{\alpha=0}=t_{1} s \pm 2 e^{\frac{t_{2}}{2}}(s+\beta)
$$

which correspond to the solutions given by the canonical coordinates $u_{1}=t_{1}+2 e^{\frac{t_{2}}{2}}$ and $u_{2}=t_{1}-2 e^{\frac{t_{2}}{2}}$ of our Frobenius manifold.

Remark 6.2 We believe that the function $F_{\alpha, \beta}^{o}$ with a correctly chosen calibration and the corresponding open descendent potential should control the genus 0 open Gromov-Witten invariants of $\mathbb{P}^{1}$, which don't have a rigorous geometric construction at the moment.

Acknowledgements We are very grateful to Paolo Rossi for informing us about his observation that solutions of the open WDVV equations correspond to flat F-manifolds of certain type. We would like to thank Alessandro Arsie, Claus Hertling, Paolo Lorenzoni, Jake Solomon and Ran Tessler for useful discussions. This project has received funding from the European Union's Horizon 2020 research and innovation programme under the Marie Skłodowska-Curie grant Agreement No. 797635. The first named author was partially supported by the Grant RFFI-18-31-20046. The second named author was partially supported by the Grant RFFI-16-01-00409.

Open Access This article is distributed under the terms of the Creative Commons Attribution 4.0 International License (http://creativecommons.org/licenses/by/4.0/), which permits unrestricted use, distribution, and reproduction in any medium, provided you give appropriate credit to the original author(s) and the source, provide a link to the Creative Commons license, and indicate if changes were made. 


\section{References}

Arsie, A., Lorenzoni, P.: Complex reflection groups, logarithmic connections and bi-flat F-manifolds. Lett. Math. Phys. 107(10), 1919-1961 (2017)

Arsie, A., Lorenzoni, P.: Flat F-manifolds, Miura invariants, and integrable systems of conservation laws. J. Integr. Syst. 3(1), xyy004 (2018)

Basalaev, A., Buryak, A.: Generalization of the Saito construction for the singularities $A$ and $D$. In preparation (2019)

Buryak, A.: Open intersection numbers and the wave function of the KdV hierarchy. Moscow Math. J. 16(1), 27-44 (2016)

Buryak, A.: Extended $r$-spin theory and the mirror symmetry for the $A_{r-1}$-singularity (2018). arXiv: $1802.07075 \mathrm{v} 2$

Buryak, A., Clader, E., Tessler, R.J.: Open $r$-spin theory and the Gelfand-Dickey wave function (2018). arXiv: $1809.02536 \mathrm{v} 1$

Buryak, A., Clader, E., Tessler, R.J.: Closed extended $r$-spin theory and the Gelfand-Dickey wave function. J. Geometr. Phys. 137, 132-153 (2019)

Buryak, A., Tessler, R.J.: Matrix models and a proof of the open analog of Witten's conjecture. Commun. Math. Phys. 353(3), 1299-1328 (2017)

Dubrovin, B.: Geometry of 2D topological field theories. Integrable systems and quantum groups (Montecatini Terme, 1993), pp. 120-348, Lecture Notes in Math., 1620, Fond. CIME/CIME Found. Subser., Springer, Berlin (1996)

Dubrovin, B.: Painlevé transcendents in two-dimensional topological field theory. The Painlevé property, CRM Ser. Math. Phys., Springer, New York, pp. 287-412 (1999)

Dubrovin, B., Liu, S.-Q., Yang, D., Zhang, Y.: Hodge integrals and tau-symmetric integrable hierarchies of Hamiltonian evolutionary PDEs. Adv. Math. 293, 382-435 (2016)

Dubrovin, B., Zhang, Y.: Frobenius manifolds and Virasoro constraints. Selecta Mathematica. N. Ser. 5(4), 423-466 (1999)

Givental, A.: Gromov-Witten invariants and quantization of quadratic Hamiltonians. Moscow Math. J. 1(4), 551-568 (2001)

Givental, A.: Symplectic geometry of Frobenius structures. Frobenius manifolds, Aspects Math., E36, Friedr. Vieweg, Wiesbaden, pp. 91-112 (2004)

Horev, A., Solomon, J.P.: The open Gromov-Witten-Welschinger theory of blowups of the projective plane (2012). arXiv:1210.4034v1

Jarvis, T.J., Kimura, T., Vaintrob, A.: Gravitational descendants and the moduli space of higher spin curves. Advances in algebraic geometry motivated by physics (Lowell, MA, 2000), pp. 167-177, Contemp. Math., 276, Amer. Math. Soc., Providence, RI (2001)

Manin, Y.: F-manifolds with flat structure and Dubrovin's duality. Adv. Math. 198(1), 5-26 (2005)

Pandharipande, R., Pixton, A., Zvonkine, D.: Tautological relations via $r$-spin structures. J. Algebra. Geometr. 28, 439-496 (2019)

Pandharipande, R., Solomon, J.P., Tessler, R.J.: Intersection theory on moduli of disks, open KdV and Virasoro (2014), arXiv:1409.2191v2

Teleman, C.: The structure of 2D semisimple field theories. Invent. Math. 188(3), 525-588 (2012)

Tessler, R.J.: The combinatorial formula for open gravitational descendents (2015). arXiv:1507.04951v3

Publisher's Note Springer Nature remains neutral with regard to jurisdictional claims in published maps and institutional affiliations. 